Article

\title{
Research on the Application of Partial Similarity for a 1-1/2 Axial Compressor
}

\author{
Hong Xie ${ }^{\mathbb{D}}$, Moru Song ${ }^{(\mathbb{D})}$ and Bo Yang * \\ School of Mechanical Engineering, Shanghai Jiaotong University, Shanghai 200240, China; \\ xiehong1211@sjtu.edu.cn (H.X.); samuel0624@sjtu.edu.cn (M.S.) \\ * Correspondence: byang0626@sjtu.edu.cn; Tel.: +86-21-3420-6871
}

Received: 18 August 2020; Accepted: 7 September 2020; Published: 9 September 2020

check for updates

\begin{abstract}
In this paper, a method based on the partial similarity principle is presented to improve the aerodynamic design with low cost and high accuracy for a 1-1/2 axial compressor. By means of this method, during the process of a similar design, the machine Mach number and flowrate coefficient are maintained. The flow similarity between the prototype and its large-scaled alternative was observed, according to a detailed analysis of flow fields of rotor and stator. As well, the relative discrepancies of isentropic efficiency and pressure ratio between two models are $1.25 \%$ and $0.4 \%$ at design point, respectively. Besides, their performance curves agreed very well in the whole operating range. Moreover, it was also found that the flow similarity between the two models can be maintained under unsteady working conditions. Thereafter, in order to investigate the impact of stability optimization method on the similarity principle, casing treatment with single circumferential groove was applied to these two models. The flow similarity was still maintained and the flowrate near the stall was reduced about $1.1 \%$ with negligible deterioration of the overall performance.
\end{abstract}

Keywords: partial similarity principle; flow similarity; stability improvement

\section{Introduction}

A high-pressure axial compressor is one of the three major parts in the state-of-the-art aero-engine, and this kind of compressor is one of the most complicated products among all compressor technology [1]. With the increasing performance and stability demands, it is vital to obtain the in-depth understanding of its internal intricate flow regimes [2]. Under the current experimental conditions, it is too difficult and hazardous to conduct experiments on the high-pressure and high-speed compressors. Consequently, low-speed and large-scale model testing developed into an achievable and reliable method, due to the benefits of low cost, better accuracy and relatively low risk [3]. The prototype can be scaled-up and its rotation speed is reduced accordingly; then thorough measurements can be realized by easily to investigate flow interactions.

The concept of low-speed model testing was first presented by Wisler in the study of exit stages in the core compressor [4]. Then, this method was applied for loss reduction and performance improvement with structural optimization [5]. Robinson improved the end-wall flow pattern in a four-stage, low-speed axial compressor with end-bent blading technology [6]. Lyes optimized the high-pressure compressor through research on blade bending and sweeping based on a low-speed model [7], which was also used for sweep and dihedral blading studies by RR Company and Cambridge University [8,9]. Besides, Boos and Lange also carried out a high loaded blading investigation on this large-scale, low-speed compressor [10,11]. Zhang to reached performance optimization of a low-speed axial rotor by three-dimensional (3D) optimization with numerical computation, and then the experiments were carried out for validation of the numerical results $[12,13]$. Zhang summarized four procedures with which to achieve the whole process of compressor scaling and performance 
optimization. The procedures included establishing a low-speed model that behaves similarly in a flow field to the high-speed compressor; detailed and accurate flow field measurement in the low-speed model; revising the design for the performance improvement; and the verification of new design methodology for the high-speed compressor at last [14]. The research above all employed the same modeling principles as that of Wisler [3]. These principles focused on some geometric parameters and aerodynamic parameters, such as the blade surface pressure and velocity distribution, and ignored the Mach number and Reynolds number.

However, the model principles mentioned above brought about some shortages. At first, the flow field is complex in 3D space, so it is not adequate to just keep the surface aerodynamic parameters the same. Then, the low-speed four-repeating-stage, studied by Wisler [3] and Zhang [14], required achieving a repeating condition for the similar inlet and outlet aerodynamic parameters. However, only the third stage can be used for the investigation, leading to wasted time and money. Additionally, this low-speed model can only be used for the investigation of a single stage, and cannot be used for multi-stage investigations. In addition, this model can be only used for the investigation at design point, but investigations at off-design points need to be similar. Besides, this kind of scaling process, based on the inverse method, is time-consuming and complicated. Finally, there is an unavoidable shortcoming of this model principle. The principle cannot be used for the transonic compressor or stage, because the shock waves' effects cannot be evaluated. Consequently, it is necessary to propose a simple, convenient, wider-in-applicability and relatively less disadvantages methodology for high-to-low-speed compressor transformation.

In recent decades, the scaling method for the low-speed model had been adopted by many researchers. The effects of Re on performance similarity were theoretically studied by Ma [15], and it has been proven that it is very important to maintain Ma in some cases $[16,17]$. In order to fulfill complete flow similarity, Ma and Re need to be identical for a high-speed compressor and low-speed model $[18,19]$. Nevertheless, it is almost impossible to maintain Re and Ma simultaneously, resulting in a performance difference between the prototype and the low-speed model. In practical applications, effects caused by changed Re can be compensated by some modifications of structural parameters [20,21]. Then, the Re could be ignored and the scaling process could be simplified accordingly, and this scaling laws can be summarized as a partial similarity principle. In this study, the partial similarity principle is innovatively used for the high-to-low transformation in the axial compressor.

The optimization of the compressor is the ultimate objective after successful establishment of the low-speed model. The stable operating range is one of the most vital objectives, and the delay of stall is an efficient method. Spike disturbance is often occurred in a low-speed compressor [22], and there are two criteria necessary for the emergence of spike disturbances, both of which are related to the tip clearance flow. One of the criteria is that the interface between the tip leakage flow and main flow aligns with the leading-edge plane [23]. Casing treatment technology has been proven to be a successful method for enhancing the stability margin of an axial compressor [24]. The interface can be delayed downstream under the impact of the casing treatment, resulting in better stall margin.

Two kinds of casing treatment are most commonly investigated. The first employs a lot of slots which are cut into the end-wall [25]. The stall margin of a compressor with slots can be improved largely, but with a large efficiency penalty [26]. The other one employs a single or several grooves which are cut into the end-wall circumferentially [27]. The stall margin improvements of a compressor with grooves are smaller than those of the first one, but the efficiency penalty is decreased [28]. Zhao concluded that the axial location had the most important effect on the stall margin improvement [29]. Houghton and Day obtained two optimum axial locations by applying a sequence of single-groove casings in two low-speed compressors-one was near the leading edge and the other near the mid-chord [30]. Du depicted that the optimal groove location in the low-speed compressor is $57 \%$ along the axial chord nearing the mid-chord [31], which coincided with the experiment results of Bailey [32].

In this study, a low-speed and large-scale axial compressor was fabricated from a high-pressure and high-speed prototype based on the partial similarity principle, the similar flow fields of which 
were validated by numerical computation. Then, in order to investigate whether the flow similarity can be contained when using the similarity principle, casing treatment with single circumferential groove was applied to these two models. Meanwhile, in this study, performance experiments were carried out to validate the reliability of the numerical method. The aim of this paper is to propose a method which can be used conveniently and quickly for a type of compressor with small dimensions and high rotation speed, rather than a certain compressor.

\section{Scaling Laws}

The design of low-speed model is different from general compressor design. The low-speed model is modeled from a high-speed prototype based on the similarity scaling laws. In general, the scaling method can be divided into exact similarity method and partial similarity method. The key difference between the two methods is whether Re remains constant or not. In this study, the Re of the prototype is calculated as Equation (1).

$$
\operatorname{Re}=\frac{U b_{2}}{v}
$$

where $v$ is the inlet kinematic viscosity.

In the practical application of the partial similarity principle, there are two dimensionless parameters that need to be kept constant [33]: the mass flow coefficient $\phi$ and the machine Mach number $M a_{U}$. The two parameters can be expressed as follows.

$$
\begin{gathered}
\phi=\frac{Q}{\pi / 4 \cdot U \cdot D_{2}^{2}} \\
M a_{U}=\frac{U}{\sqrt{\kappa R T_{0}}}
\end{gathered}
$$

The complete dimensional analysis process, based on the Buckingham-PI theorem, is described as follows. Generally, there are 7 variables overall when applying the partial similarity principle in this case, which are $\rho, u_{2}, D_{2}, P, T, t, \mu$. The length dimension $L$, mass dimension $M$ and time dimension $t$ are chosen to be the base dimensions. Simultaneously, $\rho, u_{2}, D_{2}$ are selected to be the base variables. Then, the flow characteristics and the dimensional analysis can be depicted as follows.

$$
f\left(\rho, u_{2}, D_{2}, P, T, t, \mu\right)=0,
$$

where $\mu$ is the dynamic viscosity. The deduction of similarity criterion can be described as follows.

$$
\begin{gathered}
\left\{\begin{array}{l}
\pi_{1}=\rho^{a_{1}} u_{2}^{b_{1}} D_{2}^{C_{1}} P \\
\pi_{2}=\rho^{a_{2}} u_{2}^{b_{2}} D_{2}^{C_{2}} T, \\
\pi_{3}=\rho^{a_{3}} u_{2}^{b_{3}} D_{2}^{c_{3}} t \\
\pi_{4}=\rho^{a_{4}} u_{2}^{b_{4}} D_{2}^{C_{4}} \mu
\end{array}\right. \\
\left\{\begin{array}{l}
{\left[\pi_{1}\right]=M^{0} L^{0} t^{0}=\left(M L^{-3}\right)^{a_{1}}\left(L t^{-1}\right)^{b_{1}}(L)^{c_{1}}\left(M L^{-1} t^{-2}\right)} \\
{\left[\pi_{2}\right]=M^{0} L^{0} t^{0}=\left(M L^{-3}\right)^{a_{2}}\left(L t^{-1}\right)^{b_{2}}(L)^{c_{2}}\left(L^{2} t^{-2}\right)} \\
{\left[\pi_{3}\right]=M^{0} L^{0} t^{0}=\left(M L^{-3}\right)^{a_{3}}\left(L t^{-1}\right)^{b_{3}}(L)^{c_{3}} t} \\
{\left[\pi_{4}\right]=M^{0} L^{0} t^{0}=\left(M L^{-3}\right)^{a_{4}}\left(L t^{-1}\right)^{b_{4}}(L)^{c_{4}}\left(M L^{-1} t^{-1}\right)}
\end{array}\right. \\
\left\{\begin{array}{l}
a_{1}=-1, b_{1}=-2, c_{1}=0 \\
a_{2}=0, b_{2}=-2, c_{2}=0 \\
a_{3}=0, b_{3}=1, c_{3}=-1 \\
a_{4}=-1, b_{4}=-1, c_{4}=-1
\end{array}\right.
\end{gathered}
$$




$$
\left\{\begin{array}{l}
\pi_{1}=\rho^{-1} u_{2}^{-2} D_{2}^{0} P=\frac{P}{\rho u_{2}^{2}}=\frac{\rho R T}{\rho u_{2}^{2}}=\frac{R T}{u_{2}^{2}}=\frac{1}{\kappa} \frac{\kappa R T}{u_{2}^{2}}=\frac{1}{\kappa}\left(\frac{1}{M a}\right)^{2} \\
\pi_{2}=\rho^{0} u_{2}^{-2} D_{2}^{0} T=\frac{T}{u_{2}^{2}}=\frac{1}{\kappa R} \frac{\kappa R T}{u_{2}^{2}}=\frac{1}{\kappa R}\left(\frac{1}{M a}\right)^{2} \\
\pi_{3}=\rho^{0} u_{2}^{1} D_{2}^{-1} t=\frac{u_{2} t}{D_{2}}=\frac{\frac{\pi}{4} D_{2}^{2} \cdot u_{2}}{\frac{\pi}{4} D_{2}^{2} \cdot \frac{D_{2}}{t}}=\frac{\frac{\pi}{4} D_{2}^{2} \cdot u_{2}}{Q}=\frac{1}{\phi} \\
\pi_{4}=\rho^{-1} u_{2}^{-1} D_{2}^{-1} \mu=\frac{\mu^{2}}{\rho u_{2} D_{2}}=\frac{1}{\operatorname{Re}}
\end{array},\right.
$$

Finally, the flow characteristics are summarized as Equation (8).

$$
F\left(\frac{1}{\kappa}\left(\frac{1}{M a}\right)^{2}, \frac{1}{\kappa R}\left(\frac{1}{\mathrm{Ma}}\right)^{2}, \frac{1}{\phi}, \frac{1}{\mathrm{Re}}\right)=0,
$$

Consequently, in this case, if the mass flow coefficient $\phi$ and machine Mach number $M a_{u_{2}}$ are constant and the Re is ignored, the flow similarity can be achieved in partial similarity principle.

The aerodynamic parameters at the design point of the prototype were chosen as the modeled conditions. In this study, the scaling factor is 10 , so the speed of scaled-up model is low enough according to the scaling laws. The scaled-up compressor possesses completely the same relative geometric parameters as the prototype, including solidity, aspect ratio, hub-to-tip ratio, axial-space-chord ratio, airfoil stacking, relative radial clearance, etc. In the following study, the scaling process is qualified by not only the performance curve, but also the detailed flow field characteristics of the prototype and scaled-up model. The procedure, including scaling-up and operating range optimization, is shown in Figure 1. The whole process can be divided into the scaling-up process and the improving process. Firstly, the prototype is scaled-up to be a large-scale and low-speed model, which achieves enough flow similarity with the prototype. Then, the same casing treatment is used in the prototype and large model for the performance optimization. The following sections introduce those steps one by one.

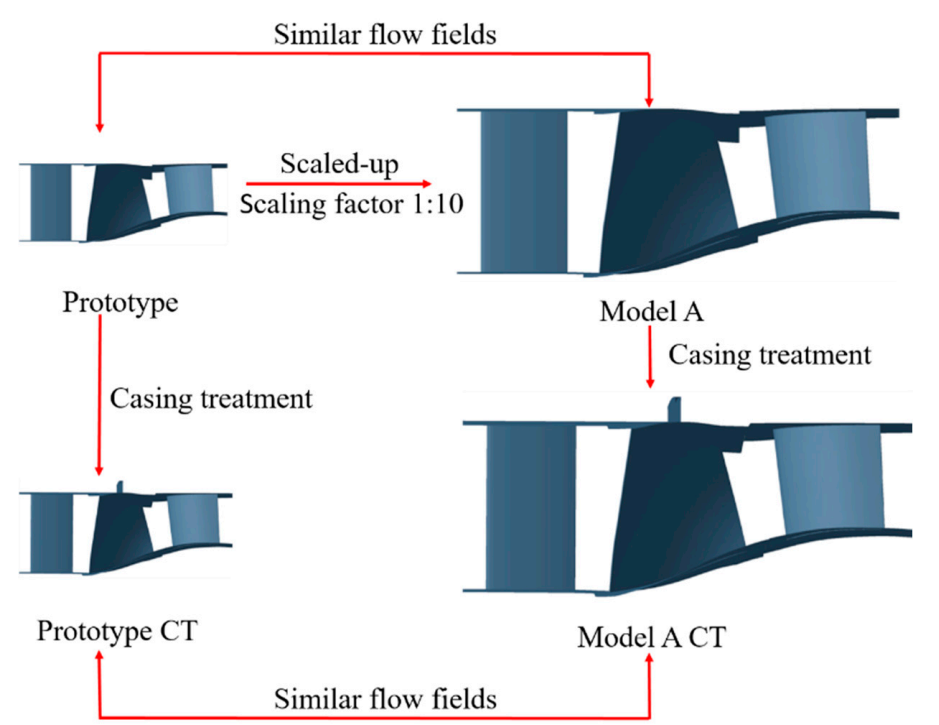

Figure 1. Scheme of performance analysis and improvement.

\section{Numerical Method}

Numerical simulations were accomplished with commercial code EURANUS, which contains steady and unsteady solvers by calculating the conservative Reynolds-averaged N-S (Navier-Stokes) equations. It discretized the N-S equations using a cell-centered finite volume formulation in space, and applied Gauss's theorem and central difference method to determine viscous flux. In order to obtain the flow regimes accurately in the flow passage and the detailed flow pattern near the casing, a second-order central spatial discretization scheme was selected to estimate the inviscid fluxes. Frozen rotor technique was employed to deal with the interface between the inlet guide vane (IGV) 
and rotor and between the rotor and stator. According to published research [34], Spalart-Allmaras's model can give fairly good results in the numerical simulation of turbomachinery. Thus, in this case, Spalart-Allmaras's model was used for turbulence simulation to evaluate the eddy viscosity [35].

Upstream and downstream, the numerical computational domain was extended, among which mesh was generated with NUMECA Auto Grid 5. The minimum grid spacing of the first layer was set to be $0.001 \mathrm{~mm}$. According to Numeca FineTurbo user guide [36], the $y+$ is recommended to be below 10 when the Spalart-Allmaras model is used. Besides, the value of $y+$ is recommended to be below 5 in some published research $[35,37]$. In this paper, the $y+$ is about 1 , which satisfies the need of the Spalart-Allmaras model. In order to analyze flow field in the tip gap, the number of total layers within tip clearance was set to be 17. A single-passage model was used in the steady computation to validate the consistency between numerical simulations and experiments. However, a single-passage model is not adequate to capture some flow characteristics of the stall inception process, such as the precursors, occurrence, evolution and initiation of the stall and the cross-passage flow. Moreover, the disturbances would propagate circumferentially when approaching the stall point, which plays a significant role in the simulation near the stall. Thus, after validating the reliability of numerical simulations, all the following numerical simulations employed the multi-passage model. As the sliding grid scheme requires two connected regions to have the same circumferential length, the same circumferential length is required for unsteady calculations. The quantities of blades can be changed based on the research of Rai [38], and the numbers of IGVs, rotors and stators were changed to 30, 30 and 30, respectively, so there were 30 passages in the full-annulus. Considering the limitations of the computing resources, a five-passage model was selected. Naturally, the performance of a five-passage model was different from that of the prototype because of the revised blade numbers, but it was considered to be enough to predict the general flow structure for the comparison among and optimization of prototype and low-speed models. The mesh numbers of single-passage and five-passage are about 3 million and 15 million, respectively, which can be seen in Figure 2. The mesh density is appropriate from the validation result of the grid independence. A three level multi-grid was adopted to accelerate the calculation.

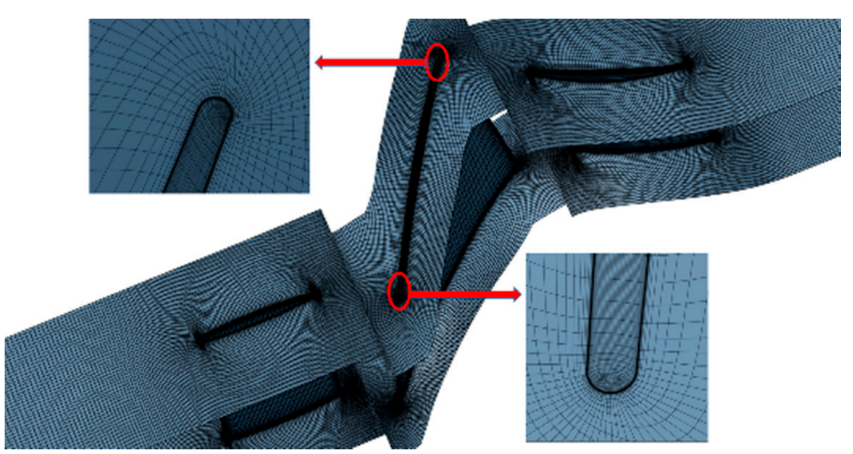

(a)

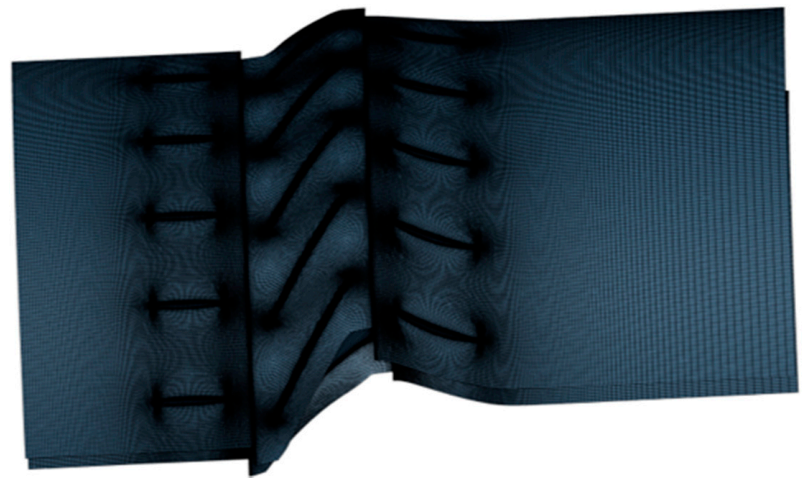

(b)

Figure 2. Mesh of computation domain: (a) single-passage model; (b) five-passage model. 
No-slip and adiabatic conditions were set at all the solid boundaries. Velocity direction, total pressure and total temperature were imposed at the inlet and average static pressure at the outlet as the boundary conditions. The back pressure was increased gradually to obtain the global stable performance maps. The last converged point was inferred to the near-stall point in this study. The steady computation results were used for the performance comparison and analysis, and the unsteady computation was also conducted to capture the unstable characteristics of the flow field. The dual-time step (DTS) method was employed to improve the time marching in the unsteady simulation. The number of angular positions was 10 in one single passage and 20 inner iterations were set for two successive computations, which is enough to get detailed unsteady information.

\section{Results and Analysis}

\subsection{Evaluation of the Numerical Method}

For the validation of the numerical method, the test was carried out in the 1-1/2 stage axial subsonic compressor in Shanghai Jiao Tong University, as shown in Figure 3 [39]. The blade numbers were 32, and 29 and 37 for the inlet guide vane (IGV), rotor and stator respectively. The section profile of all blades is NACA0012. Hub/tip ratio (ratio of the diameter of blade hub to the diameter of blade tip) was 0.76. The rotational speed was 12,000 rpm, with which the tip Mach number was less than 0.9 at design point. The height of tip clearance was $1 \%$ chord length at the blade tip. To acquire the aerodynamic parameters of inlet and outlet, temperature sensors and pressure sensors were arranged at the upstream (plane A) and downstream (plane D) areas, as shown in Figure 3a. On plane A, two static pressure probes and two five-hole total pressure probes (accuracy $0.02 \%$ ) were installed to obtain inlet static pressure and total pressure, respectively. One five-point temperature probe (accuracy $0.2 \%$ ) was set to measure the inlet total temperature. The same types sensors were equipped on the plane $D$ to obtain outlet pressure and temperature. In this case, the absolute measurement errors of total pressure and temperature were about $0.02 \mathrm{Kpa}$ and $0.5 \mathrm{~K}$, respectively. The measurement error of prototype-stage isentropic efficiency was about $1.5 \%$. The overall performance of the prototype was acquired with the adjustment of throttle area at outlet. A flowmeter was placed upstream of IGV to measure the flow rate. The geometric parameters of prototype are listed in Table 1.

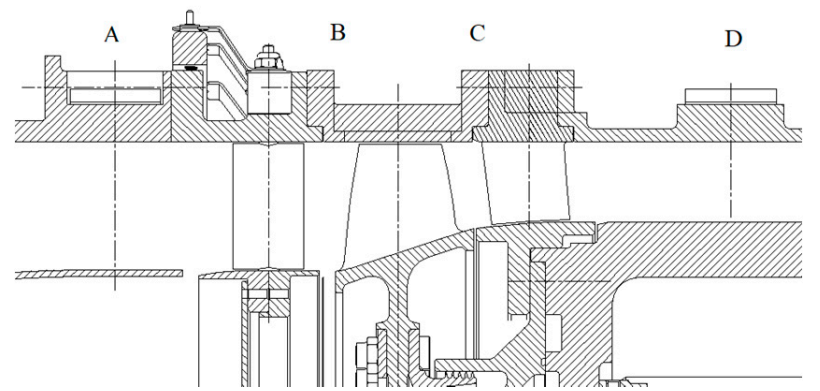

(a)

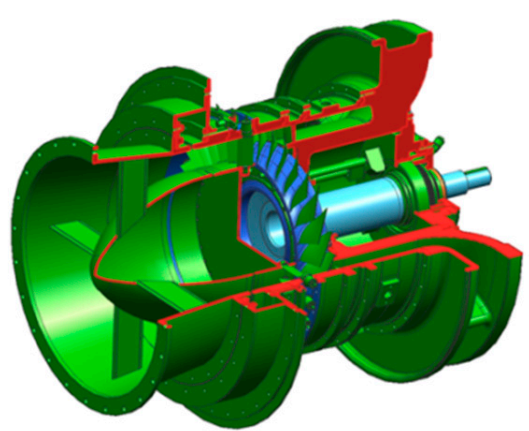

(b)

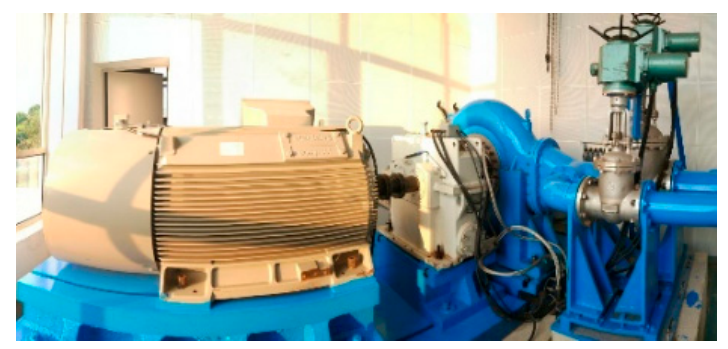

(c)

Figure 3. Test facility: (a) meridional plane of prototype; (b) 3D model of prototype; (c) test rig. 
Table 1. Design parameters of the prototype.

\begin{tabular}{ccc}
\hline Parameters & Unit & Value (Prototype) \\
\hline Diameter, D2 & $\mathrm{mm}$ & 370 \\
Hub/tip ratio & - & 0.76 \\
Blade height & $\mathrm{mm}$ & 56 \\
Tip clearance, $\varepsilon$ & $\mathrm{mm}$ & 0.65 \\
Rotor blade chord length, b2 & $\mathrm{mm}$ & 65 \\
$\varepsilon / b_{2}$ & - & $1 \%$ \\
Rotation speed, $\mathrm{N}$ & $\mathrm{rpm}$ & 12,000 \\
\hline
\end{tabular}

The experiment and numerical performance versus normalized flow rate are shown in Figure 4. Squares, triangles and circles were applied to make a distinction among the performance curves of experiment results and numerical results of single-passage and five-passage, respectively. The upper lines depict the isentropic efficiency characteristic and the lines below depict the pressure ratio characteristic. In this case, the isentropic efficiency $\eta_{s}$ is calculated as Equation (10).

$$
\eta_{s}=\frac{\left(P_{1} / P_{0}\right)^{\frac{\kappa-1}{\kappa}}-1}{T_{1} / T_{0}-1}
$$

where $\kappa$ is the Adiabatic exponent, $P_{0}$ the inlet total pressure, $P_{1}$ the outlet total pressure, $T_{0}$ the inlet total temperature and $T_{1}$ the outlet total temperature.

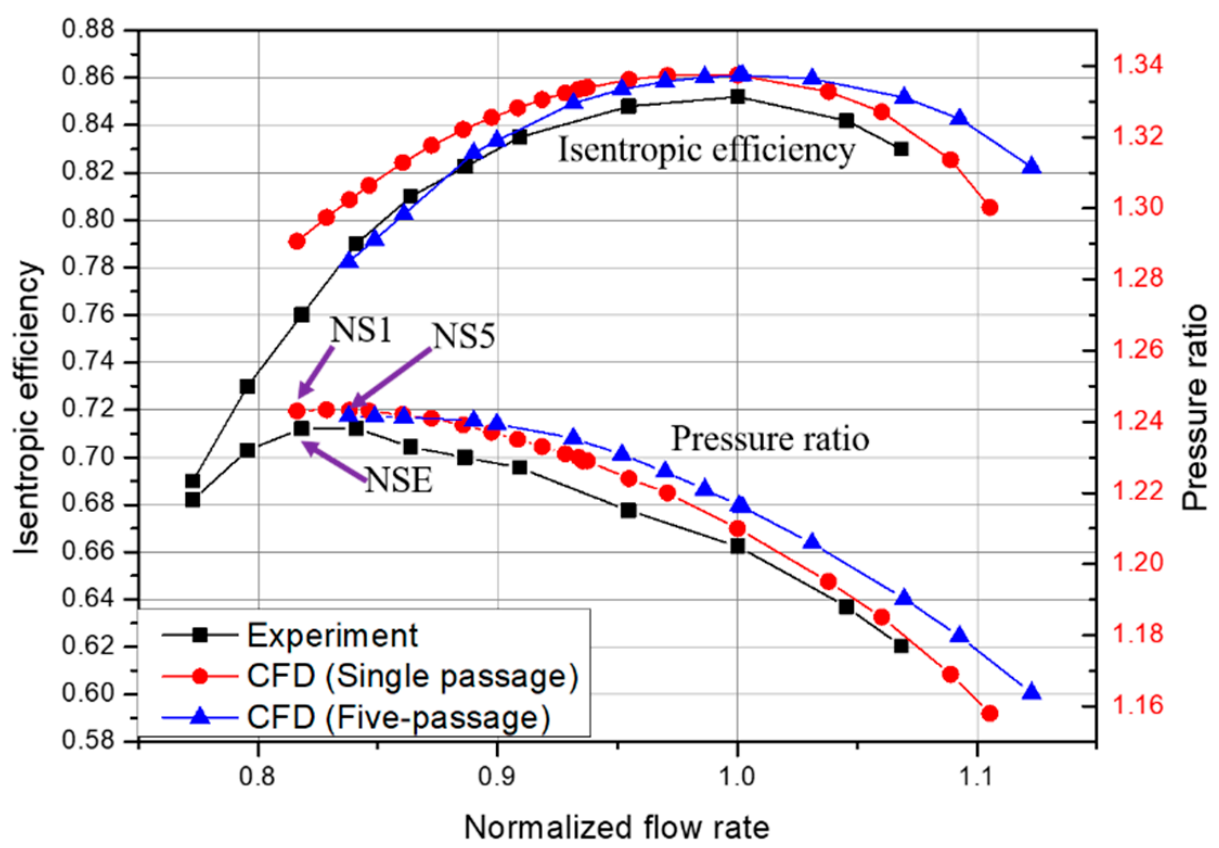

Figure 4. Overall performances of prototype stages.

Three points are labeled in Figure 4 for depicting the near-stall points conveniently. NSE represents the last point of the characteristic at minimum normalized flow rate Q/Qref $=0.77$ in experiment, NS1 and NS5 represent the solution limit under the steady computation of single-passage and five-passage, respectively. The computation results of single-passage and experiment results correspond well with each other. The discrepancy at design point is less than $1.5 \%$, and the tendencies of the curves agree well. The major difference at the off-design points is that the computation results overestimate the limit flow rate about $4.2 \%$; that is because of the limitation of the steady simulation. However, the NS1 is very close to the NSE, which means that the steady simulation can exactly capture the point near the stall. The errors exist between the curves of single-passage and five-passage because of the changed 
blade number. The five-passage model shows better performance in a relatively large flow rate, and the single-passage model shows a better stall margin. In generally, the steady simulation was adequately accurate to obtain the performance and the point near the stall. The five-passage model was used for the follow-up study to capture the circumferential flow characteristics, and the five-passage high-speed model was set to be the prototype accordingly.

\subsection{Performance Analysis of the Scaled-Up Compressors}

\subsubsection{Comparison of the Prototype and Scaled-Up, Low-Speed Model A at Design Point}

The low-speed model A was scaled-up with the scaling factor 1:10 from the prototype geometrically, and the five-passage model was too. Model A maintained the machine Mach number and mass flow coefficient and kept the inlet and outlet parameters. The geometric and aerodynamic parameters of the prototype and model A are listed in Table 2.

Table 2. Design parameters of prototype and compressor A.

\begin{tabular}{cccc}
\hline Parameters & Unit & Value (Prototype) & Value (Model A) \\
\hline Inlet total pressure, $\mathrm{P}_{0}$ & $\mathrm{kPa}$ & 101.025 & 101.025 \\
Inlet total temperature, $\mathrm{T}_{0}$ & $\mathrm{~K}$ & 284 & 284 \\
Design Mass flow rate, $\mathrm{Q}_{d}$ & $\mathrm{Kg} / \mathrm{s}$ & 6.1 & 610 \\
Diameter, $\mathrm{D}_{2}$ & $\mathrm{~mm}$ & 370 & 3700 \\
Tip clearance, $\varepsilon$ & $\mathrm{Mm}$ & 0.65 & 6.5 \\
Rotor blade chord length, $\mathrm{b}_{2}$ & $\mathrm{~mm}$ & 65 & 650 \\
$\varepsilon / b_{2}$ & - & $1 \%$ & $1 \%$ \\
Design rotational speed, $\mathrm{N}$ & $\mathrm{rpm}$ & 12,000 & 1200 \\
Peripheral velocity of impeller, $\mathrm{U}$ & $\mathrm{m} / \mathrm{s}$ & 232 & 232 \\
Reynolds number, Re & & $1.05 \times 10^{6}$ & $1.05 \times 10^{6}$ \\
Mass flow coefficient & - & 0.2446 & 0.2446 \\
Working fluid & - & $\mathrm{Air}$ & $\mathrm{Air}$ \\
Adiabatic index, $\kappa$ & - & 1.4 & 1.4 \\
\hline
\end{tabular}

The distributions of averaged circumferential static pressure coefficient, $C_{p}$, at five spanwise points of the rotor and stator for the prototype and model $\mathrm{A}$ at design point are shown in Figures 5 and $6 . C_{p}$ is defined as

$$
C_{p}=\frac{P}{P_{\text {total, }, \text { in }}}
$$

where $P$ and $P_{\text {total, in }}$ denote the surface static pressure and the total pressure at inlet. The static pressure distributions of numerical results of prototype and model A are shown in Figure 5. Overall, the distributions of static pressure of rotor between prototype and model A are almost exactly the same in the whole flow passage. However, there is still small a difference at mid-span, which will be analyzed next. The flow field near the hub and the casing reached good flow similarity, which was not realized by the former studies. The distributions of static pressure of stators also agree well, as shown in Figure 6. Nevertheless, the flow similarity of the stator is not as good as that of the rotors. Moreover, the similarity of the stator over 50\% spanwise was worse than that of rotor. Besides, it can be seen that the static pressure rise of stator of model A was larger than that of prototype at all spanwise values.

The comparisons of averaged circumferential values of aerodynamic parameters along the blade height of rotor and stator are shown in Figures 7 and 8. In general, the tendencies of inlet and outlet relative flow angles of the rotor match well. The distributions of flow turning angle near the end-wall are nearly the same. However, the discrepancy of occurs from $5 \%$ to $95 \%$ blade height, for which the maximum is about 1.5 degrees. The good agreement also appears at the axial velocity distributions at inlet and outlet of rotor. However, the outlet axial velocity of model A is a little smaller than that of the prototype at the same region as the flow turning angle distribution. It can be inferred that the 
decreased axial velocity attributed the decreasing turning angle, which may be related to the variation of Re. The flow angle and axial velocity distributions of stators still agree well for the prototype and model A, though the error of outlet flow angle is bigger than that of rotor, which may not only be affected by the enlarged Re but the upstream effects of the rotor.

Averaged circumferential isentropic efficiency of the rotor and loss coefficient distributions of rotor and stator along blade height are shown in Figures 9 and 10, respectively. The total pressure loss coefficients of rotor $\omega_{R}$ and stator $\omega_{S}$ are expressed as:

$$
\begin{gathered}
\omega_{R}=\left(P_{0 w}-P_{1 w}\right) /\left(P_{0 w}-P_{0}\right) \\
\omega_{S}=\left(P_{1 t}-P_{2 t}\right) /\left(P_{1 t}-P_{1}\right)
\end{gathered}
$$

where $P_{0}$ is the inlet static pressure of the rotor, $P_{0 w}$ is the average inlet relative total pressure of the rotor, $P_{1 w}$ is the average outlet relative total pressure of the rotor, $P_{1}$ is the inlet static pressure of the stator, $P_{2}$ is the average outlet absolute total pressure of the stator and $P_{1 t}$ is the average inlet absolute total pressure of the stator. The efficiency of model $\mathrm{A}$ is improved from $10 \%$ to $85 \%$ blade height, and the loss is reduced slightly accordingly. The efficiency and loss curves are approaching. There are two regions with obvious error that can be observed in the loss curves of stators; one is near the hub end-wall and one is from $70 \%$ to $100 \%$ blade height. The discrepancy may have resulted from the different impact on the downstream stator, caused by the wake flow, leakage flow and main flow. This impact varied with the varied wake flow, leakage flow and main flow along the radial direction, because of the different absolute values of blade height and axial gap between the rotor and stator. Thus, the difference in the upper parts of the downstream stators was brought about. Besides, the difference near the hub end-wall may be relative to the different friction loss, caused by the different boundary effects because of different Re and blade height.
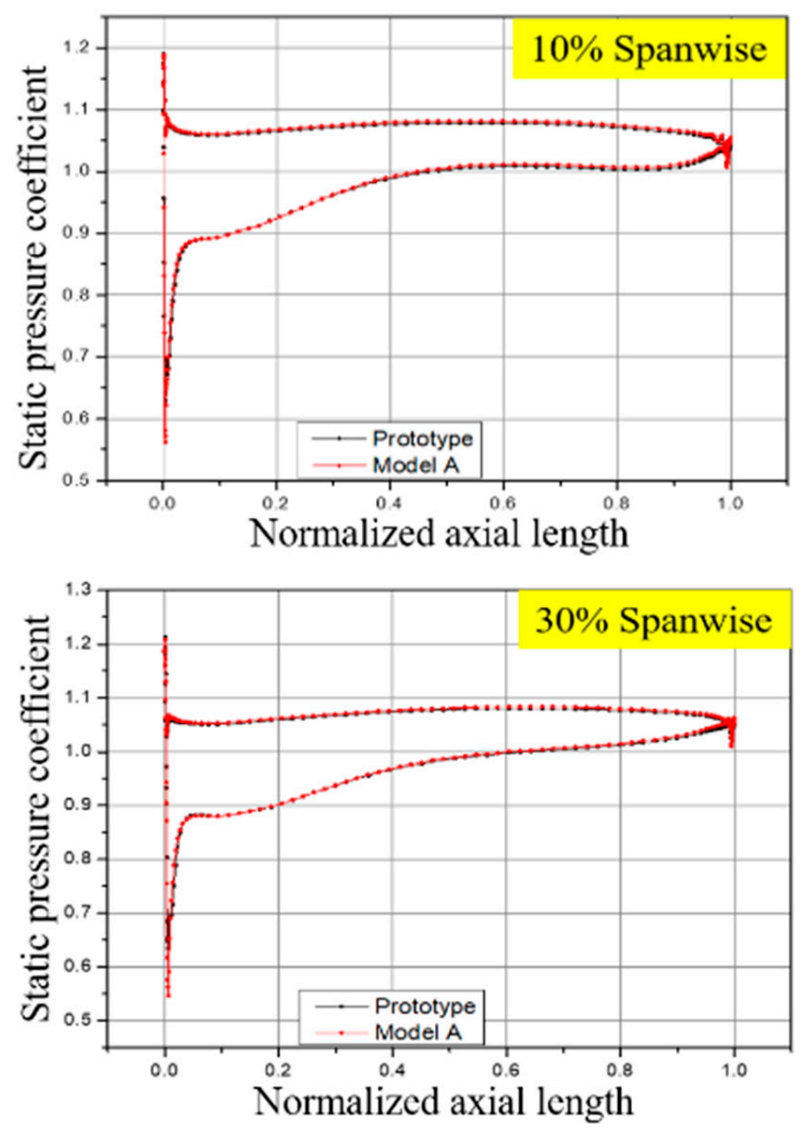

Figure 5. Cont. 

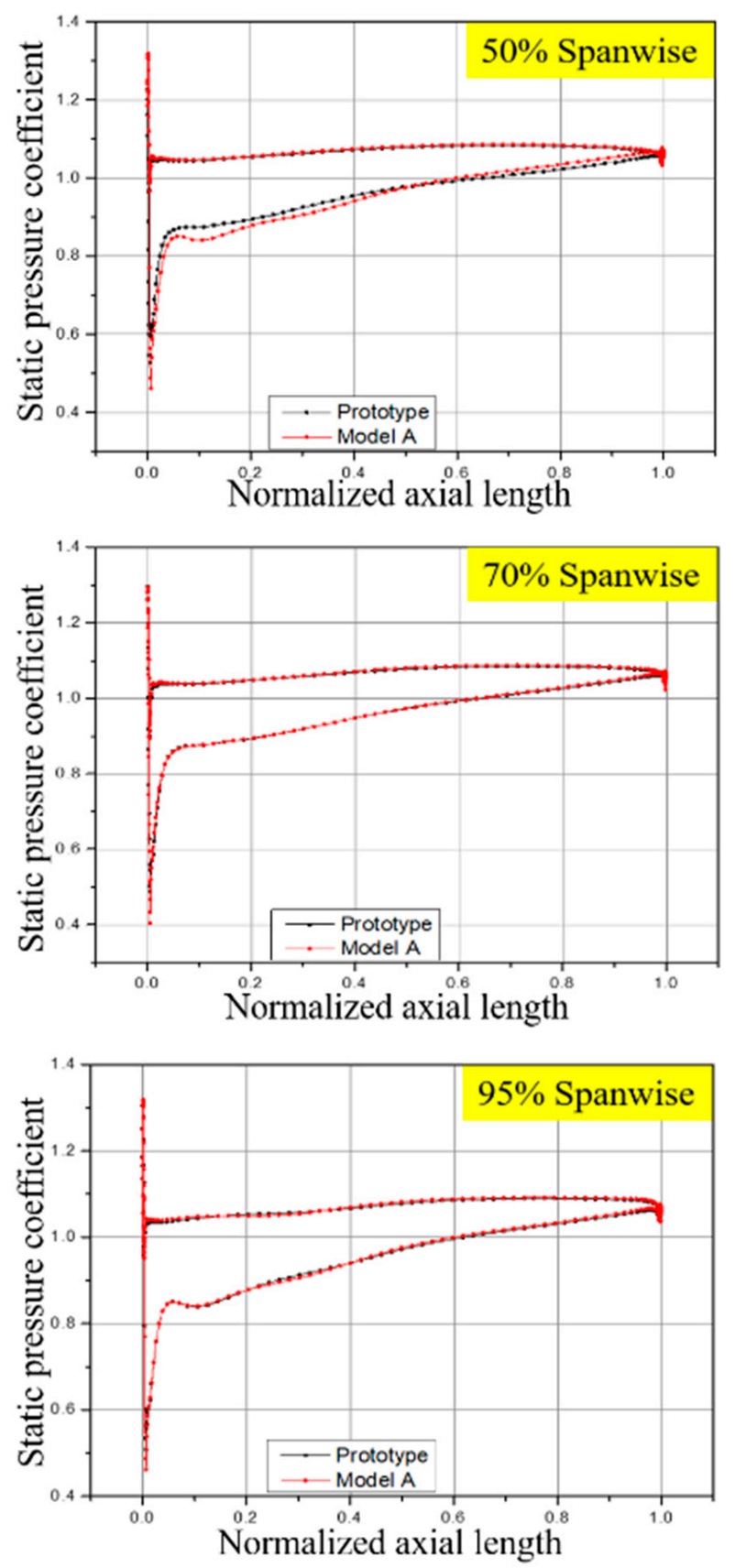

Figure 5. Static pressure distributions of the rotor.

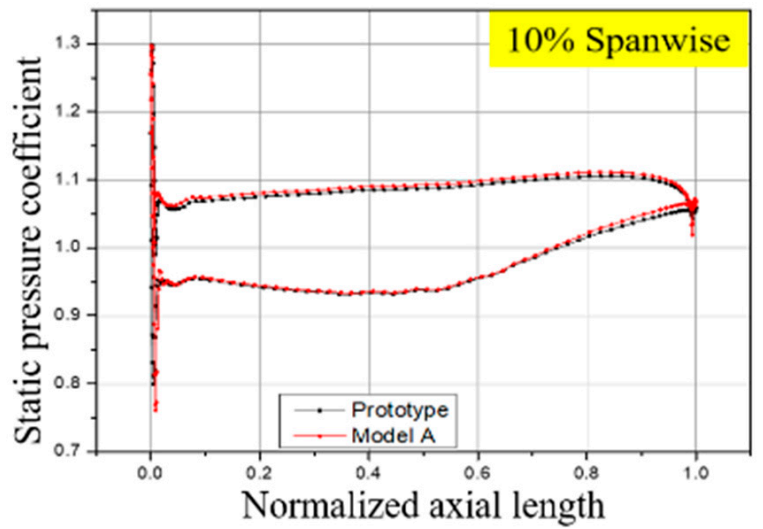

Figure 6. Cont. 

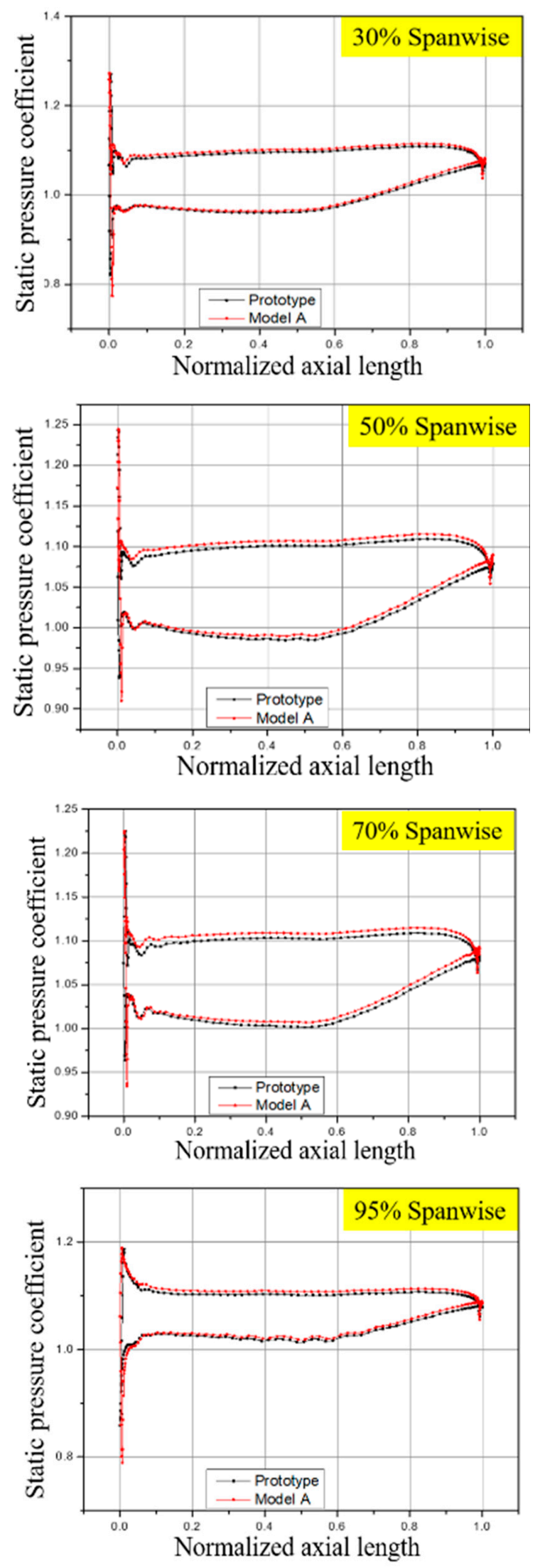

Figure 6. Static pressure distributions of the stator. 

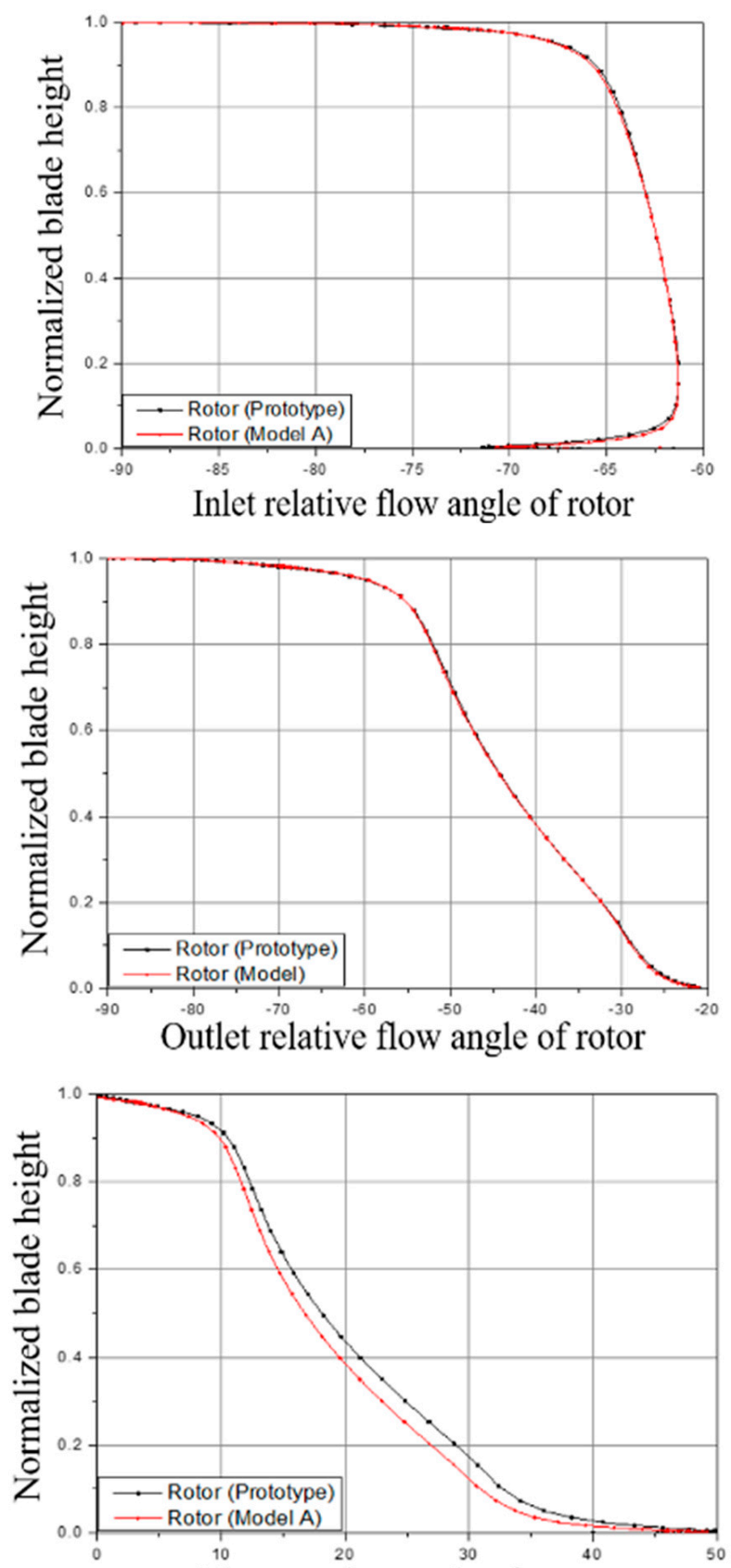

Flow turning angle of rotor

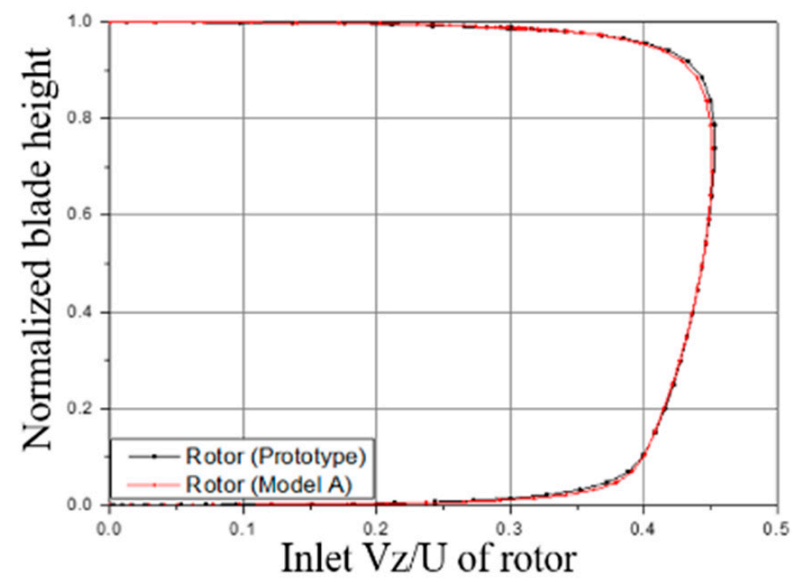

Figure 7. Cont. 


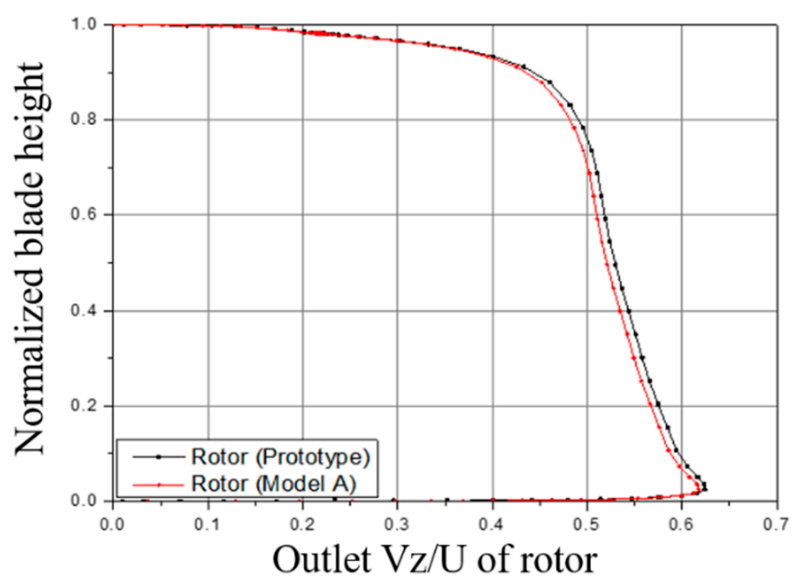

Figure 7. Averaged circumferential values of aerodynamic parameters of the rotor.
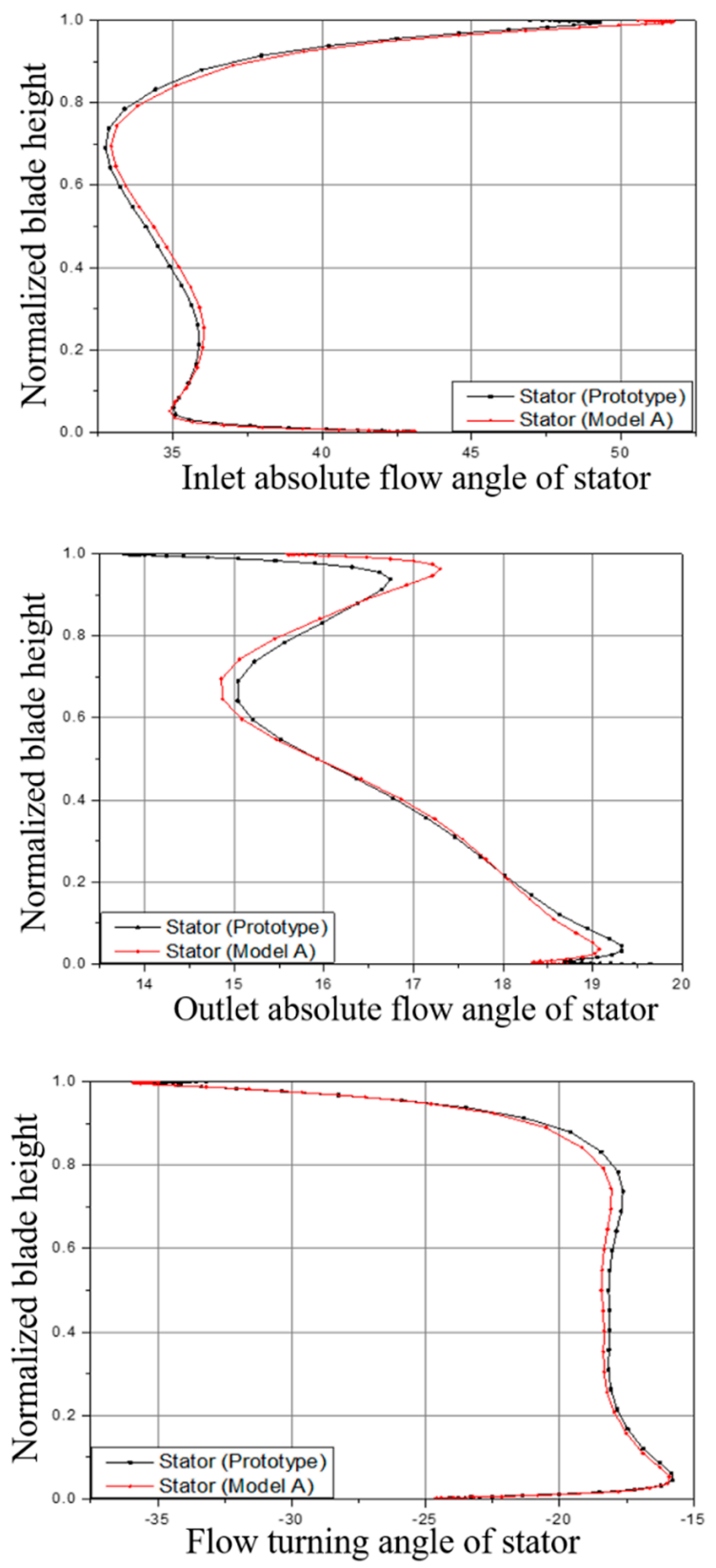

Figure 8. Cont. 

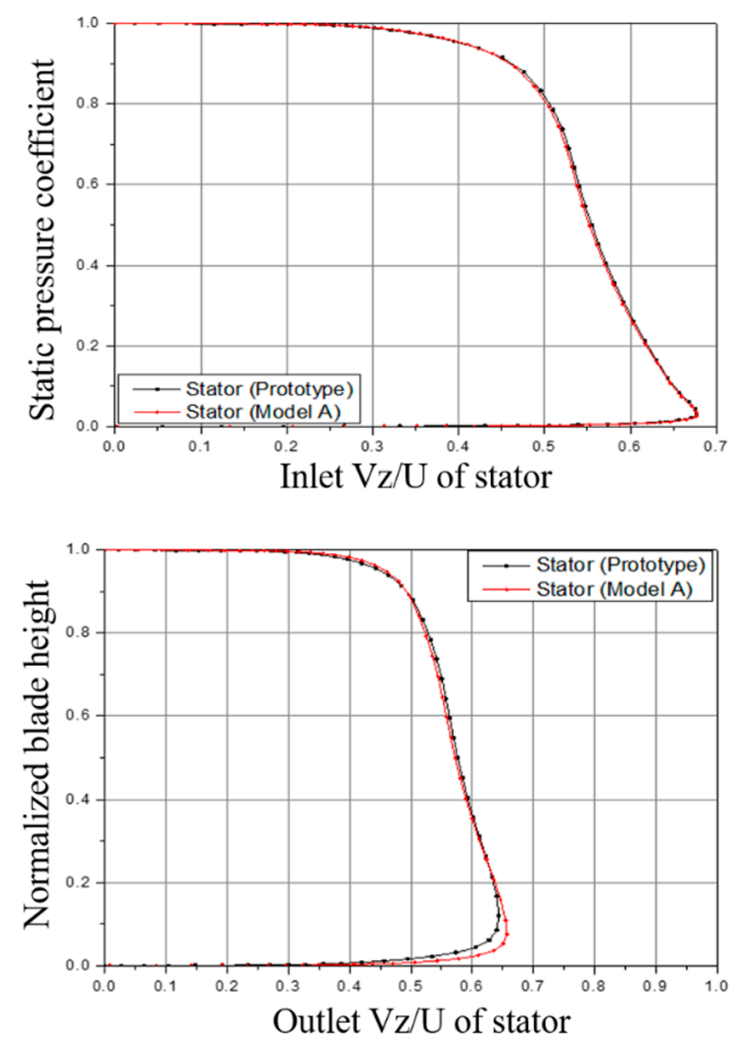

Figure 8. Averaged circumferential values of aerodynamic parameters of the stator.

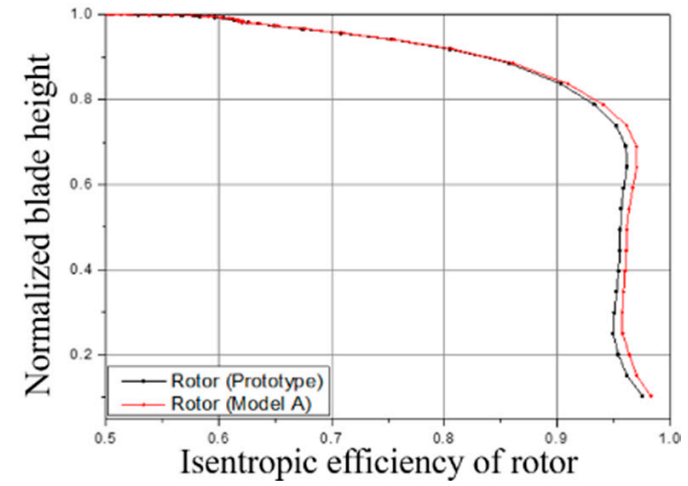

Figure 9. Isentropic efficiency of the rotor.

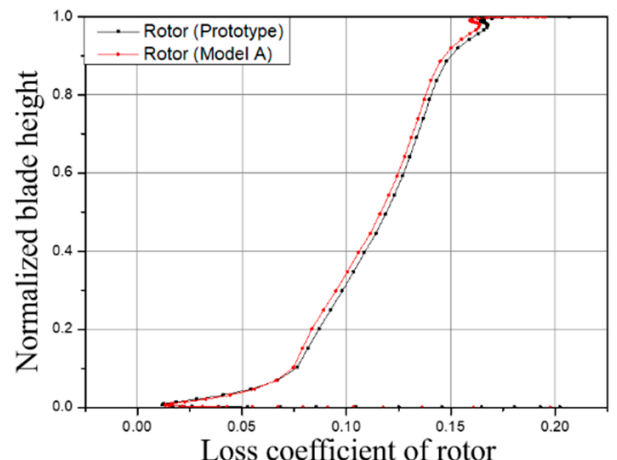

(a)

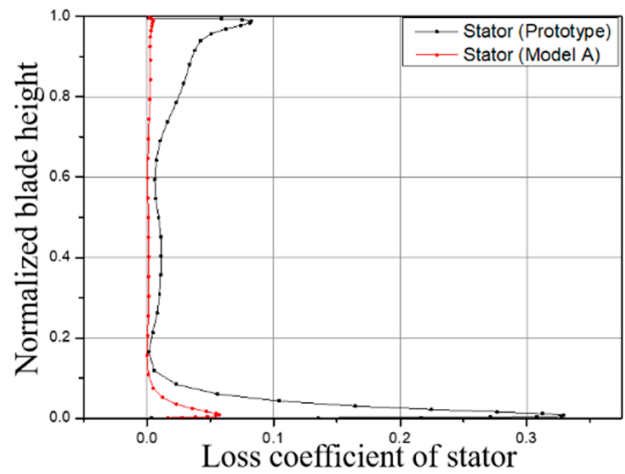

(b)

Figure 10. Loss coefficient distributions of the rotor and stator: (a) loss coefficient of rotor; (b) loss coefficient of stator. 
According to the comparison and analysis of this section, the low-speed model was successfully fabricated based on the scaling laws. Exact flow similarity was achieved in the rotor and stator, although there was a little error. It is very convenient to realize the scaling process without a complicated iterative process based on the complex inverse method, and the flow similarity of the rotor and stator is reached at the same time. After the successful process at design point, the study on the off-design followed, which was for discussing the flow similarity in the whole operating range.

\subsubsection{Comparison of the Prototype and Scaled-Up, Low-Speed Model A in Operating Conditions}

The performance comparison between the prototype and model A is shown as a function of normalized mass flow rate in Figure 11. The tendency of performance curves agrees well for the prototype and model A under the operating flow rate. The discrepancies of efficiency and pressure were less than $1.5 \%$ and $0.5 \%$, and they were only $1.25 \%$ and $0.4 \%$ at design point, respectively. It was proven that the flow similarity is not only achieved at design point but for the whole operating flow rate based on the partial similarity principle. However, the flow rate near the stall of model A (the normalized flowrate at this point is namely, NSA) was about $4.2 \%$ less than that of prototype (the normalized flowrate at this point is namely, NSA), meaning that the stability is worsened when conducting the scaling-up process.

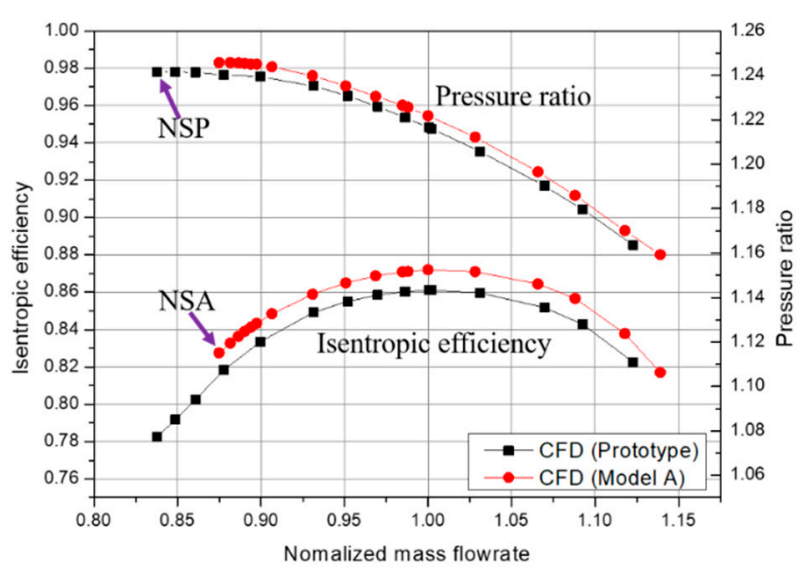

Figure 11. Performance in the whole operating range.

The static pressure and entropy distributions near the casing ( $99.5 \%$ spanwise) of model A at NSA, for prototype at NSP, and for the prototype at NSA, are compared in Figure 12. The red dotted line is static pressure and it is used to detect the trajectory of tip clearance vortex [40]. One of the characteristics for spike disturbance initiating rotating stall is that the interface, where the oncoming and tip leakage flows meet, is aligned to the leading-edge plane. In other words, the tip leakage flow spilled below the blade tip [32]. As shown in Figure 12a, the tip leakage flow of model A at NSA nearly spills below the blade tip, and this flow phenomenon can be also observed in the prototype at NSP. Furthermore, the flow field between model and prototype is similar, so it can be inferred that the flow similarity is maintained near the stall point, and the type of stall inception is also the same. The tip leakage vortex of prototype migrates downstream at NSA, near the stall point of model A. It illustrates that the scaling-up model promotes the tip leakage vortex moving upstream, which results in rotating stalling before the prototype. The entropy distribution can be applied for distinguishing the interface between main flow and tip leakage flow [31]. The black dashed line, where the entropy increases severely, describes the location of the interface. The black line is successive and parallel to the leading-edge plane at NSA in model A and at NSP in the prototype, meaning the inception of stalling. The interface of prototype at NSA is still in the passage, corresponding with the analysis on the static pressure distribution.

Based on the above sections, the high-speed compressor is successfully transformed to the low-speed, large-scale compressor in the whole operating range, although there are some tiny errors. 
These tiny errors may be caused for several reasons, such as the changed Re, the variations of absolute values of geometric parameters and so on. The further research about diminishing the errors was the content of the next study to achieve further flow similarity. It is worth noting that almost complete flow similarity was achieved under steady conditions, as shown in Figures 5, 7,9 and 10. Then, it was necessary to study whether the flow similarity was contained in the unsteady state when using the scaling laws, because some important flow characteristics are closely related to the unsteady condition.

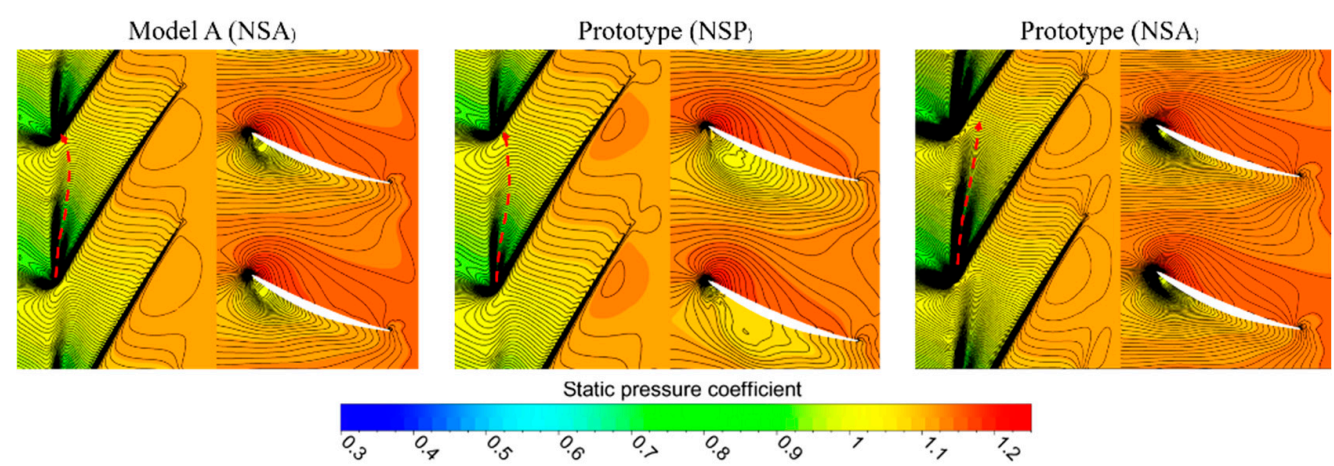

(a)

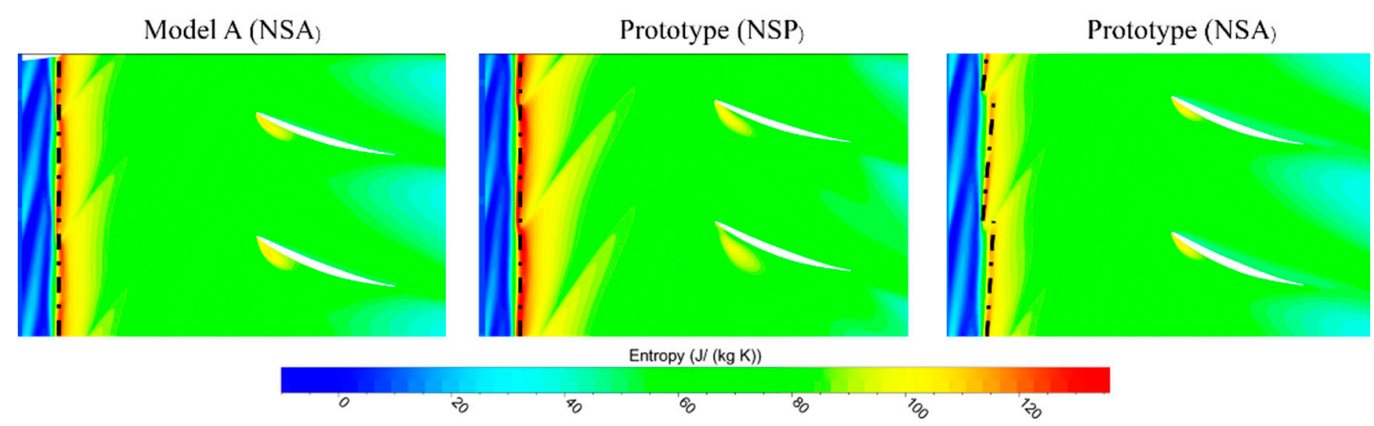

(b)

Figure 12. Comparisons of static pressure and entropy distributions at $99.5 \%$ spanwise: (a) static pressure distribution; (b) entropy distribution.

\subsubsection{Comparison of the Prototype and Scaled-Up, Low-Speed Model A under Unsteady Condition}

The flow similarity between the high-speed prototype and low-speed large-scale model A is realized under steady condition. The transient normalized relative axial velocity contours at $99.5 \%$ and 97\% spanwise of prototype and model A at design point are shown in Figure 13. It can be observed that the flow field within the tip clearance and near the casing is stable, and there is not migration of tip leakage vortex. The velocity at $99.5 \%$ spanwise of model A is a little larger than that of prototype along the trajectory of leakage vortex, while it is opposite at $97 \%$ spanwise. Yet, on the whole, the evolution of the flow field for prototype is similar to that of and model, implying that the flow similarity is preserved under the unsteady condition at design point. The transient characteristics of velocity at $99.5 \%$ and $97 \%$ spanwise between prototype and model $\mathrm{A}$ at the near-stall point are compared in Figure 13.

The instantaneous velocity contours near the stall are shown in Figure 14. The fluctuation of the red dotted curve, detecting the trajectory of tip leakage vortex, indicates that the tip leakage vortex is unstable. The vortex is generated at the leading edge of the blade, and then migrates circumferentially, which can be deduced from the region depicted with $\mathrm{A}$ and $\mathrm{B}$. When the vortex impinges the adjacent blades, its intensity is decreased, and the secondary leakage even multi-leakage appears. The red ellipse also presents the movement of the vortex. Again, the unstable characteristics of the prototype and model near the stall are similar, demonstrating that the unsteady flow features are maintained in the design and off-design operating conditions. 
99.5\% Spanwise of prototype (Design point)

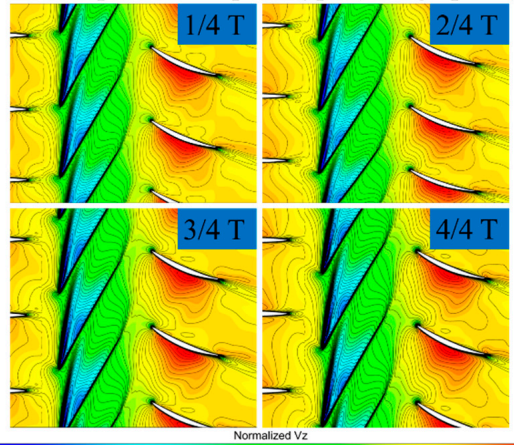

${ }_{3}$

97\% Spanwise of prototype (Design point)

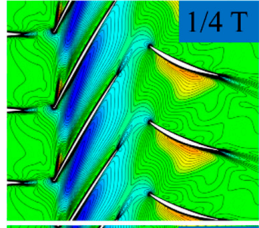

$-1$

$-$

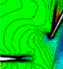

$=\sqrt{1}$

\section{य)}
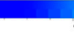
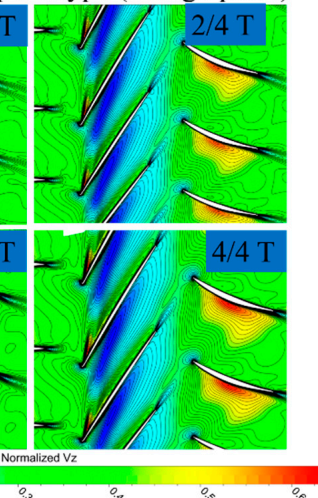

99.5\% Spanwise of Model A (Design point)

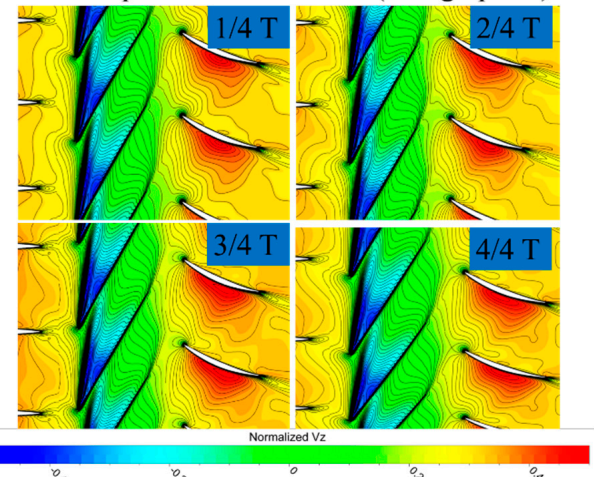

97\% Spanwise of Model A (Design point)

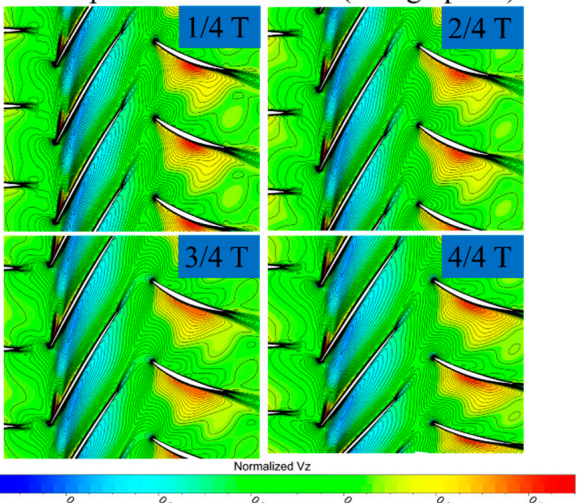

Figure 13. Transient axial relative velocity of the prototype and model A at $99.5 \%$ and $97 \%$ spanwise at design point.


Figure 14. Transient axial relative velocity of the prototype and model A at $99.5 \%$ and $97 \%$ spanwise near the stall. 


\subsection{Stability Improvement through Casing Treatment}

In this study, the single circumferential groove was applied as the casing treatment technology. The location and geometric parameters, and the mesh of the flow passage with groove of model A are shown in Figure 15, and the mesh number of the groove was about 5 million, which is fine enough to provide grid independence.
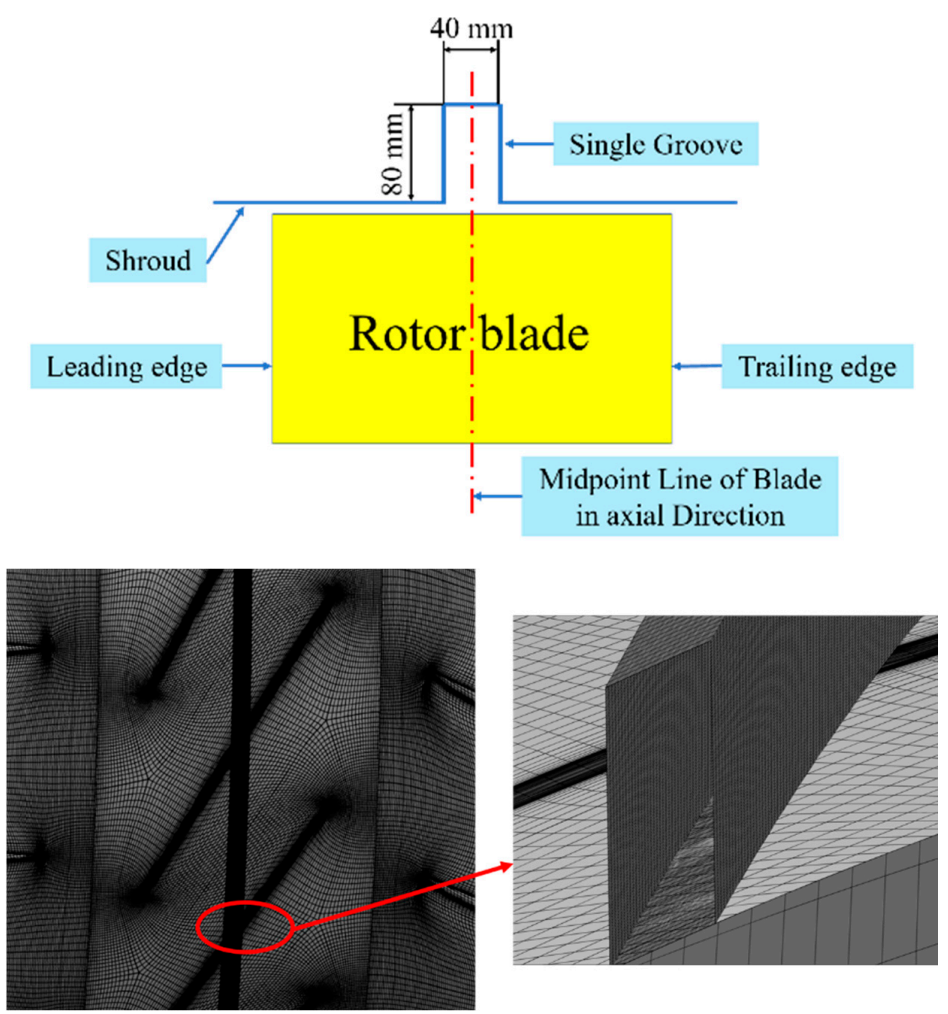

Figure 15. Sketch map of groove geometry and mesh.

The characteristics of the smooth solid wall and the grooved casing treatment are shown in Figure 16. The model A with a groove is named model A CT, and NSACT means the near-stall point of model A CT. The efficiency and pressure ratio of model A were decreased about $0.4 \%$ and $0.3 \%$ at design point. The reduction of performance decreased along the direction of small flowrate and increased towards the large flowrate.

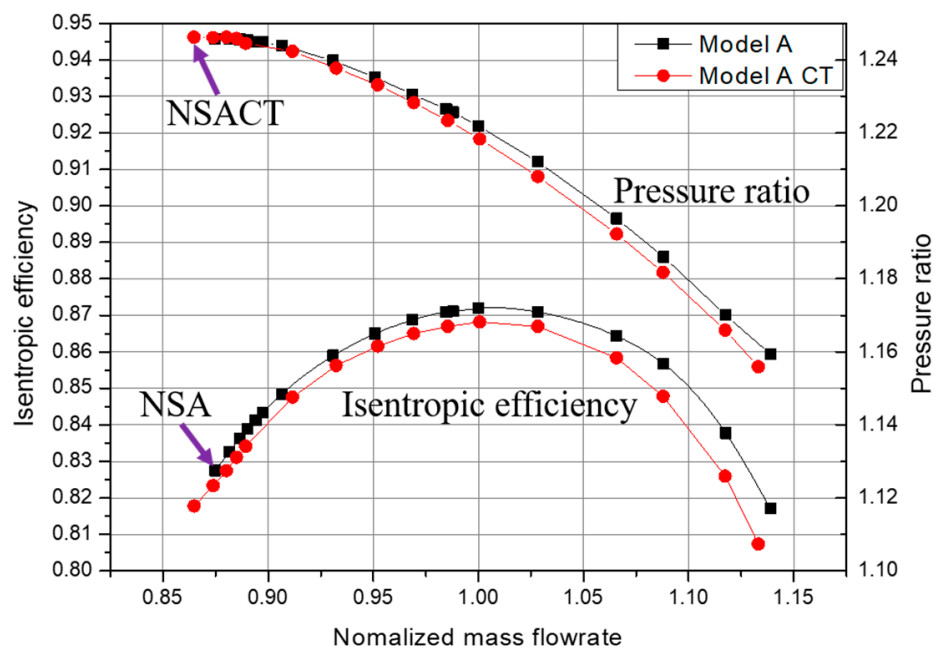

Figure 16. Pressure ratio and isentropic efficiency of a smooth solid wall and grooved casing treatment. 
The reason for the decrease can be explained in Figure 17, which shows the apparent increasing entropy around and downstream the region existing groove. The two black lines added in this figure represent the upstream and downstream edges of groove. Besides, it can be observed that the impact of groove not only exists in the tip clearance but in the region near the casing. However, the solution limit near the stall is obviously moved toward the small flowrate, elucidating that the stability improvement of model A was achieved. The reduction of the flowrate near the stall of the prototype was about $1.2 \%$.

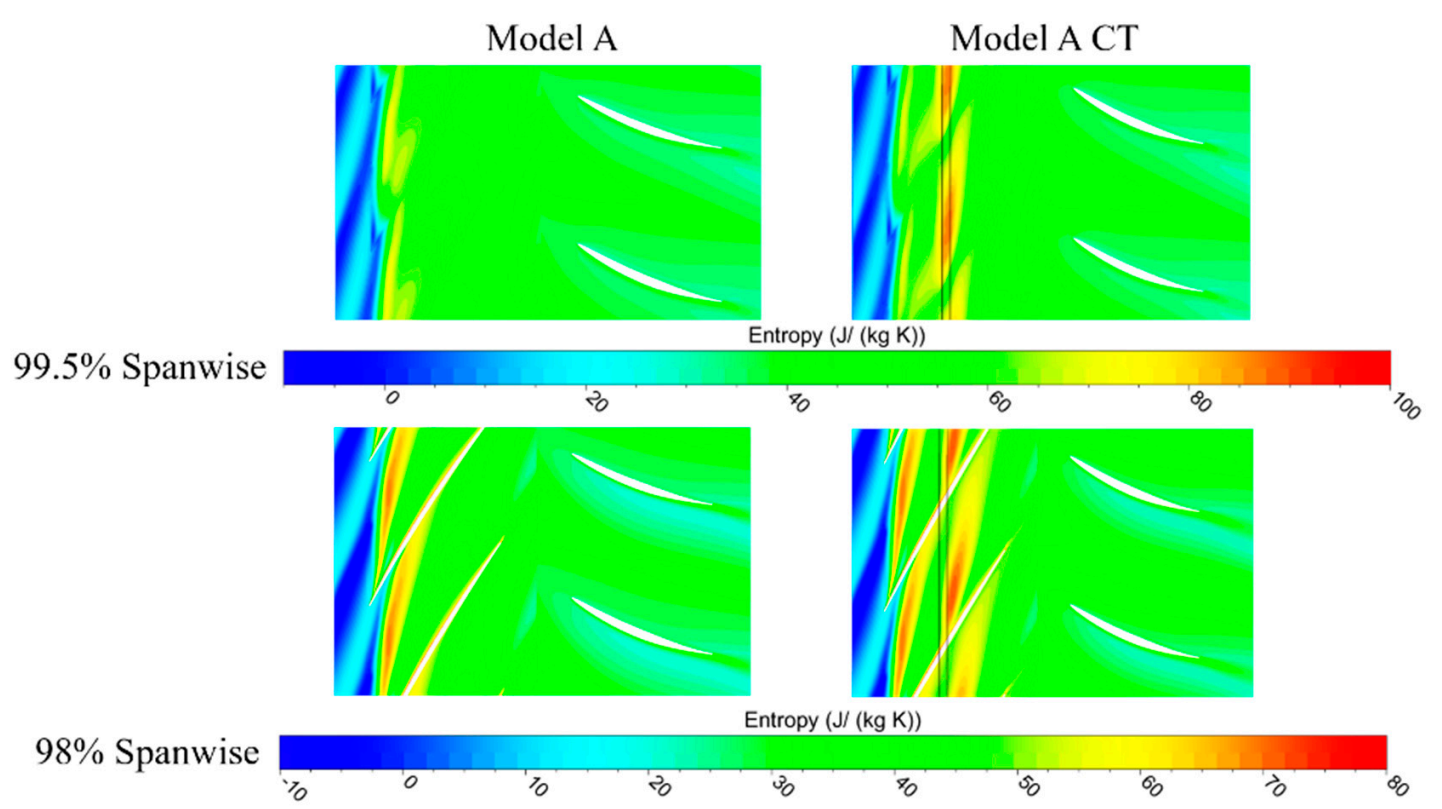

Figure 17. Entropy distributions at $99.5 \%$ and $98 \%$ spanwise.

The stability improvement of the prototype was the ultimate object of this research, so the single circumferential groove was also applied to the prototype. The location of the groove was the same as for model A CT, and the geometric parameters were scaled down by the scaling factor 1:10 accordingly. The prototype applying the groove was named prototype CT, and NSPCT means the near-stall point of model A CT. The simulation results for the prototype and prototype CT are shown in Figure 18. The efficiency and pressure ratio of prototype decreased about $0.54 \%$ and $0.35 \%$ at design point-a little more than for model A, and the tendencies of the curves are similar to those of model A. The reduction of the flowrate of the prototype was $1.1 \%$, which is very close to that of model A.

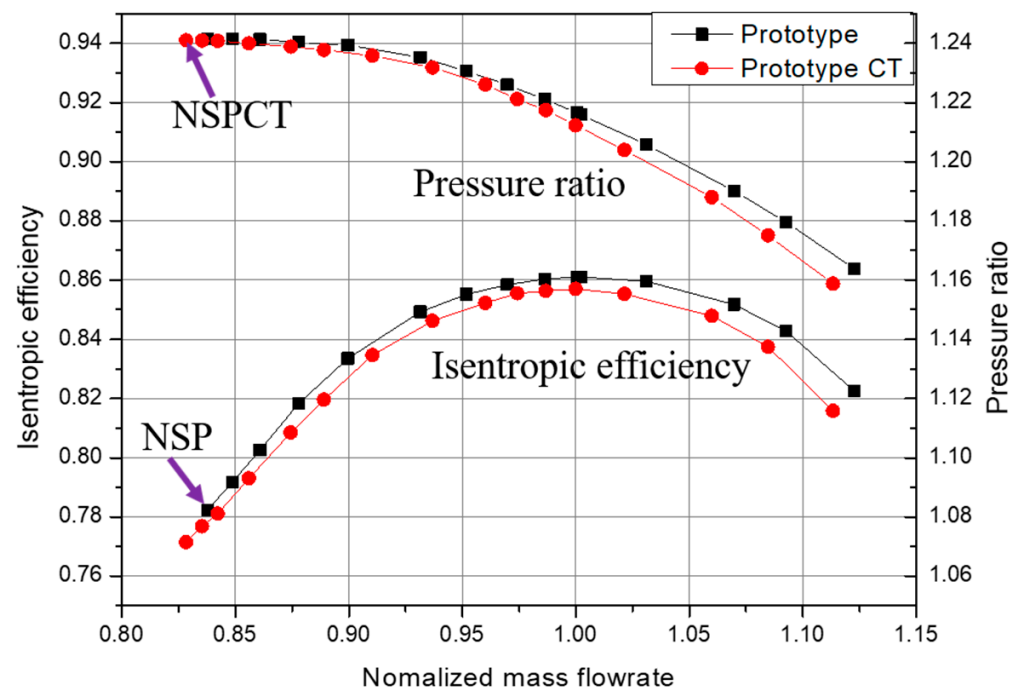

Figure 18. Pressure ratio and isentropic efficiency of the smooth solid wall and grooved casing treatment. 
As shown in Figure 19, the entropy also increased apparently around and downstream the region existing groove. The higher entropy distribution around groove of prototype explain the larger performance decrease of prototype. Furthermore, the entropy distribution of prototype CT is similar to that of model A CT, indicating that the effect of groove on the two models is similar and the flow similarity is maintained. The mechanism of stability improvement applying the circumferential groove has been elucidated clearly in many publications [27-29], which is outside the scope of this study, so it will not be covered in this paper. The flow similarity between prototype and model A prove that the research of the casing treatment on the model A can be used for the prototype.

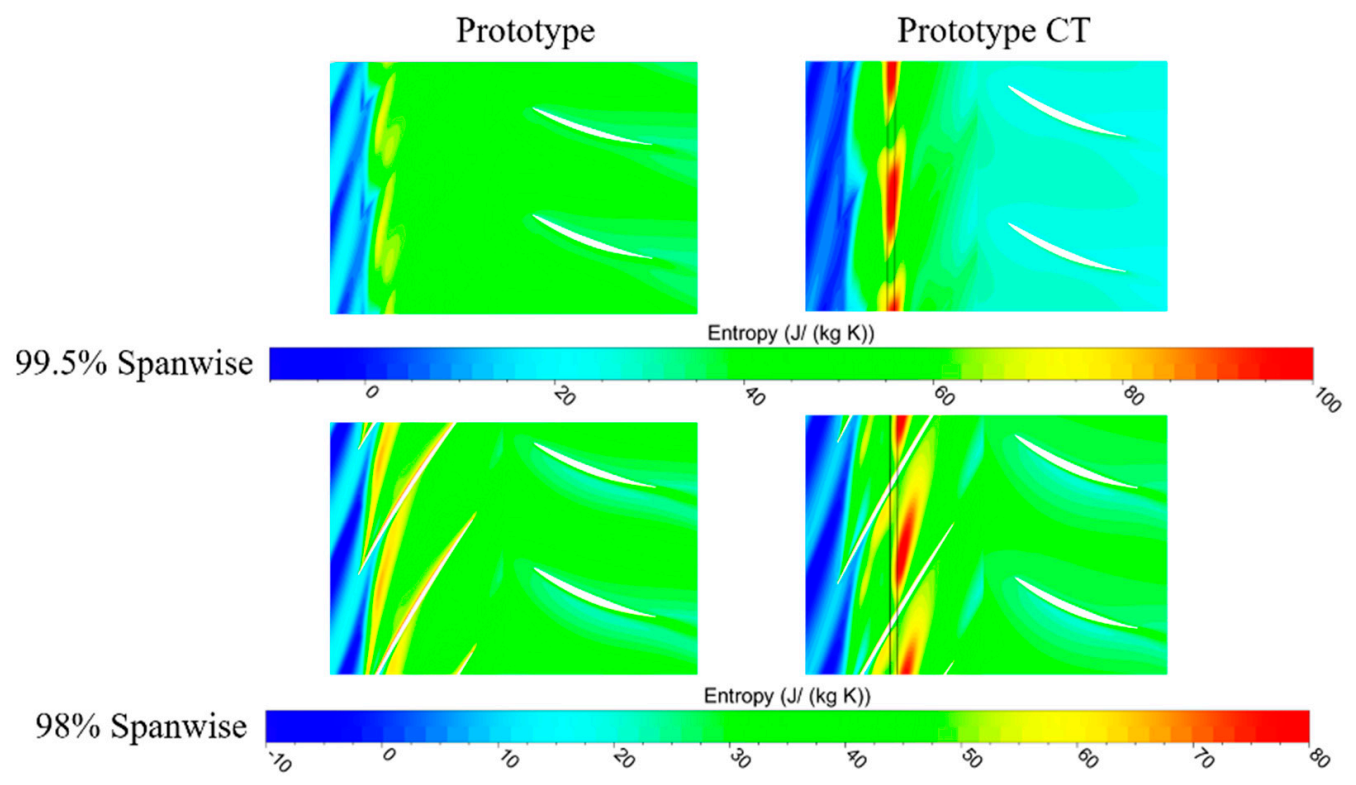

Figure 19. Entropy distributions at $99.5 \%$ and $98 \%$ spanwise.

\section{Discussion}

In this study, the flow similarity at the design and off-design points has been achieved well, except that there is a little distinction in the solution limit flowrate near the stall. Thus, further flow similarities will be focused on in the following research, such as the geometric corrections, including the value of tip clearance, the axial gap between rotor and stator, etc. Besides, the casing treatment is one of the effective technologies for stability and performance improvement; there are many other technologies can be used. Furthermore, the detailed measurement of aerodynamic parameters through establishing low-speed large-scale test facility will be carried out after the adequate flow similarity is achieved.

Some comments on the effect of Reynolds number on aerodynamic performance are significant. When scaling-up the prototype, the Re increases as the absolute dimensions of the prototype are enlarged, and the relationship of Re between the prototype and scaled model has been presented in the research of Perter [41]. The friction coefficient value of scaled-up model is decreased with increasing Re, which leads to better efficiency. Nevertheless, the decrease of friction coefficient is finite. The friction coefficient is determined not only by Re but also by the relative height of the roughness of the surface, which is limited. However, CFD computations in this paper were carried out for the cases of smooth surfaces and the roughness of the surface was neglected in this paper. Besides, the displacement thickness of the boundary layer is also effected by the changed Re [42]. Therefore, further investigation is necessary and will be discussed in future research.

\section{Conclusions}

In this paper, a method based on the partial similarity principle is proposed. In practical applications, this method can be used conveniently and quickly for the transformation of a high-speed, 
small compressor. In our case, a 1-1/2 axial compressor has been scaled up to be a low-speed, large-scale model, based on the high-to-low-speed transforming methodology-the partial similarity principle. Thereafter, casing treatment was used for the stability improvement and the flow similarity still remained. Numerical simulations were used for the analysis of the scaled-up process and the stability optimization process. In addition, experimental tests were carried out for the validation of simulation results. On the basis of studies, the following conclusions were drawn.

- The high-speed compressor was successfully transformed to be a low-speed, large-scale model based on the partial similarity principle, which keeps the Ma and flowrate coefficient constant and neglects the effects of Re. This principle is simple and convenient compared to the conventional low-speed model methodology based on the inverse method.

- The surface aerodynamic parameters of the rotor and stator are maintained well. The maximum deviations of isentropic efficiency and pressure between prototype and model A were $1.5 \%$ and $0.5 \%$, respectively. The deviations were only $1.25 \%$ and $0.4 \%$ at design point. The flow fields were similar in the whole operating range. The flow similarity and the type of stall inception were retained at the near-stall point. The error of the solution limit flowrate near the stall was about $4.2 \%$, which may have been caused by the changed Re and absolute value of geometric value. Additionally, further flow similarity, through geometric corrections to compensate for the effects of changed Re and dimensions, will be researched in next study.

- A single circumferential groove was used as the casing treatment technology. The stability was improved with a negligible performance penalty. The reductions of the near-stall flowrate of the prototype and model were $1.1 \%$ and $1.2 \%$, respectively. The similar flow fields and performance indicated that the flow similarity was kept when casing treatment was employed. The flow similarity was maintained not only under steady condition but unsteady condition, according to the unsteady simulation results.

Author Contributions: Conceptualization, H.X. and B.Y.; methodology, H.X.; software, M.S.; validation, H.X., M.S.; formal analysis, H.X.; investigation, H.X.; resources, H.X., M.S.; data curation, H.X.; writing-original draft preparation, H.X.; writing-review and editing, B.Y.; visualization, H.X.; supervision, B.Y.; project administration, H.X.; funding acquisition, B.Y. All authors have read and agreed to the published version of the manuscript.

Funding: This research was funded by the National Science and Technology Major Project (2017-V-0012-0064).

Conflicts of Interest: The authors declare no conflict of interest.

\section{References}

1. Koff, B.L. Gas Turbine Technology Evolution: A Designers Perspective. J. Propuls. Power 2004, 20, 577-595. [CrossRef]

2. Massardo, A.; Satta, A.; Marini, M. Axial Flow Compressor Design Optimization: Part II-Throughflow Analysis. ASME. J. Turbomach. 1990, 112, 405. [CrossRef]

3. Wisler, D.C. Loss Reduction in Axial-Flow Compressor Through Low-Speed Model Testing. ASME. J. Eng. Power 1985, 107, 90. [CrossRef]

4. Wisler, D.C. Core Compressor Exit Stage Study; NASA CR No. 135391; NASA Lewis Research Center: Cleveland, OH, USA, 1977; Volume 1.

5. Wisler, D.C. Improving Compressor and Turbine Performance through Cost-Effective Low-Speed Testing. In Proceedings of the ISABE 14th International Symposium on Air-Breathing Engines, Florence, Italy, 5-10 September 1999. ISABE 99-7073.

6. Robinson, C.J. Endwall Flows and Blading Design for Axial Flow Compressors. Ph.D. Thesis, Cranfield University, Cranfield, UK, 1991.

7. Lyes, P.A. Low Speed Axial Compressor Design and Evaluation; High Speed Representation and Endwall Flow Control Studies. Ph.D. Thesis, Cranfield University, Cranfield, UK, 1999.

8. Gallimore, S.J.; Bolger, J.J; Cumpsty, N.A.; Taylor, M.J.; Wright, P.I.; Place, J.M. The Use of Sweep and Dihedral in Multistage Axial Flow Compressor Blading-Part I: University Research and Methods Development. ASME J. Turbomach. 2002, 124, 521-532. [CrossRef] 
9. Gallimore, S.J.; Bolger, J.J.; Cumpsty, N.A.; Taylor, M.J.; Wright, P.I.; Place, J.M. The Use of Sweep and Dihedral in Multistage Axial Flow Compressor Blading-Part II: Low and high-speed designs and test verification. ASME J. Turbomach. 2002, 124, 533-541. [CrossRef]

10. Boos, P.; Mockel, H.; Henne, J.M.; Seimeler, R. Flow Measurement in a Multistage Large Scale Low Speed Axial Flow Research Compressor. In Proceedings of the ASME, Turbo Expo: Power for Land, Sea, and Air, Stockholm, Sweden, 2-5 June 1998; ASME Paper No. 98-GT-432. p. V001T01A104. [CrossRef]

11. Lange, M.; Vogeler, M.K.; Mailach, R.; Elorza-Gomez, S. An experimental Verification of a New Design for Cantilevered Stators with Large Hub Clearance. ASME J. Turbomach. 2013, 135, 041022. [CrossRef]

12. Zhang, C.K.; Hu, J.; Wang, Z.Q.; Yin, C.; Yan, W. Numerical Study on Three-Dimensional Optimization of a Low-Speed Axial Compressor Rotor. In Proceedings of the ASME, Turbo Expo: Power for Land, Sea, and Air, Dusseldorf, Germany, 16-20 June 2014; ASME Paper No. GT2014-26431. p. V02BT45A016. [CrossRef]

13. Zhang, C.K.; Hu, J.; Wang, Z.Q.; Li, J. Experimental Investigations on Three-Dimensional Blading Optimization for Low-Speed Model Testing. ASME J. Gas. Turbines Power 2016, 138, GTP-15-1570. [CrossRef]

14. Zhang, C.K.; Hu, J.; Wang, Z.Q.; Gao, X. Design Work of a Compressor Stage Through High-To-Low Speed Compressor Transformation. ASME J. Gas. Turbines Power 2014, 136, 064501. [CrossRef]

15. Ma, Y.; Xi, G. Effects of Reynolds Number and Heat Transfer on Scaling of a Centrifugal Compressor Impeller. In Proceedings of the ASME, Turbo Expo: Power for Land, Sea, and Air, Glasgow, UK, 14-18 June 2010; ASME Paper No. GT2010-23372. pp. 565-572. [CrossRef]

16. Zhu, C.; Qin, G. Performance Prediction of Centrifugal Compressor Based on Performance Test, Similarity Conversion and CFD Simulation. Int. J. Fluid Mach. Syst. 2012, 5, 38-48. [CrossRef]

17. Florian, F.; Peter, J.; Hoiger, F. On the Scaling of Aeroelastic parameters for High Pressure Applications in Centrifugal Compressors. In Proceedings of the ASME, Turbo Expo: Power for Land, Sea, and Air, Seoul, Korea, 13-17 June 2016; ASME Paper No. GT2016-57409. p. V07BT34A019. [CrossRef]

18. Asad, M.S.; William, K.; George, W.K., Jr. Reconsideration of the Fan Scaling Laws: Part I-Theory. In Proceedings of the ASME/JSME 2003 4th Joint Fluids Summer Engineering Conference, Honolulu, HI, USA, 6-10 July 2003; ASME Paper No. FEDSM2003-45414. pp. 1307-1315. [CrossRef]

19. Asad, M.S.; William, K.; George, W.K., Jr. Reconsideration of the Fan Scaling Laws: Part II-Applications. In Proceedings of the ASME/JSME 2003 4th Joint Fluids Summer Engineering Conference, Honolulu, HI, USA, 6-10 July 2003; ASME Paper No. FEDSM2003-45418. pp. 1329-1336. [CrossRef]

20. Zhu, N.G.; Xu, L.; Chen, M.Z. Similarity transformation for compressor blading. ASME J. Turbomach. 1991, 114, 561-568. [CrossRef]

21. Xie, H.; Yang, B.; Zhang, S.; Song, M. Performance Analysis and Improvement of a Centrifugal Compressor Based on Partial Similarity Principle. ASME J. Eng. Gas. Turbines Power 2020, 142, 05012. [CrossRef]

22. Tan, C.S.; Day, I.; Morris, S.; Wadia, A. Spike-Type Compressor Stall Inception, Detection, and Control. Annu. Rev. Fluids Mech. 2010, 42, 275-300. [CrossRef]

23. Vo, H.D.; Tan, C.S.; Greitzer, E.M. Criteria for Spike Initiated Rotaing Stall. ASME J. Turbomach. 2008, 130, 011023. [CrossRef]

24. Du, J.; Gao, L.P.; Li, J.C.; Lin, F.; Chen, J.Y. Initial selection of groove location combination for multi-groove casing treatment. J. Mech. Sci. Technol. 2015, 30, 697-704. [CrossRef]

25. Seitz, P.A. Casing Treatment for Axial Flow Compressors. Ph.D. Thesis, Cambridge University, Cambridge, UK, 1999.

26. Wilke, I.; Kau, H.-P.A. Numerical Investigation of the Flow Mechanisms in a High Pressure Compressor Front Stage with Axial Slots. ASME J. Turbomach. 2004, 126, 339-349. [CrossRef]

27. Osborn, W.M.; Lewis, G.W.; Heidelberg, L.J. Effects of Several Porous Casing Treatments on Stall Limit and Overall Performance of an Axial Compressor Rotor; NASA Report TN 6537; National Aeronautics and Space Administration: Washington, DC, USA, 1971.

28. Fujita, H.; Takatta, H. A study on configurations of casing treatment for axial flow compressors. JSME Bull. 1983, 27, 1675-1681. [CrossRef]

29. Zhao, S.F.; Lu, X.G.; Zhu, J.Q.; Zhang, H.W. Investigation for the Effects of Circumferential Grooves on the Unsteadiness of Tip Clearance Flow to Enhance Compressor Flow Instability. In Proceedings of the ASME, Turbo Expo: Power for Land, Sea, and Air, Glasgow, UK, 14-18 June 2010; ASME Paper No. GT2010-22652. pp. 269-277. [CrossRef] 
30. Houghton, T.; Day, I. Enhancing the Stability of Subsonic Compressors Using Grooves. ASME J. Turbomach. 2011, 133, 021007. [CrossRef]

31. Du, J.; Gao, L.P.; Li, J.C.; Lin, F.; Chen, J.Y. The Impact of Casing Groove Location on Stall Margin and Tip clearance Flow in a Low-Speed Axial Compressor. ASME J. Turbomach. 2016, 138, 121007. [CrossRef]

32. Bailey, E.E. Effect of Grooved Casing Treatment on the Flow Range Capability of a Single-stage Axial-slow Compressor; NASA Report TM 2459; National Aeronautics and Space Administration: Washington, DC, USA, 1972.

33. Dufour, G.; Carbonneau, X.; Cazalbou, J.; Chassaing, P. Practical Use of Similarity and Scaling Laws for Centrifugal Compressor Design. In Proceedings of the ASME, Turbo Expo: Power for Land, Sea, and Air, Barcelona, Spain, 8-11 May 2006; ASME paper No. GT2006-91227. pp. 1131-1140. [CrossRef]

34. Ning, F.; Xu, L. Numerical investigation of transonic compressor rotor flow using an implicit 3D flow solver with one-equation Spalart-Allmaras turbulence model. In Proceedings of the ASME, Turbo Expo: Power for Land, Sea, and Air, New Orleans, LA, USA, 4-7 June 2001; ASME paper No. 2001-GT-0.59. p. V001T03A054. [CrossRef]

35. Spalart, P.R.; Allmaras, S.R. A one equation turbulence model for aerodynamic flows. In Proceedings of the 30th Aerospace Sciences Meeting and Exhibit, Reno, NV, USA, 6-9 January 1992. AIAA paper. [CrossRef]

36. Numeca. Numeca FINETurbo User Guide, Version 11.1; NUMECA: Beijing, China, 2017.

37. Assam, A.; Narayan Kalkote, N.; Sharma, V.; Eswaran, V. An Automatic Wall Treatment for Spalart-Allmaras Turbulence Model. ASME J. Fluids Eng. 2018, 140, 061403. [CrossRef]

38. Rai, M.M. Three-Dimensional Navier-Stokes Simulations of Turbine Rotor-Stator Interaction. ASME J. Turbomach. 1987, 112, 377-384. [CrossRef]

39. Song, M.R.; Yang, B.; Dong, G.M.; Liu, X.L.; Wang, J.Q.; Xie, H.; Lu, Z.H. Research on Accuracy of Flowing Field Based on Numerical Simulation for Tonal NoisePrediction in Axial Compressor. In Proceedings of the ASME, Turbo Expo: Power for Land, Sea, and Air, Oslo, Norway, 11-15 June 2018. ASME Paper No. GT2018-76382. [CrossRef]

40. Yoon, Y.S.; Song, S.J.; Shin, H.W. Influence of Flow Coefficient, Stagger Angle, and Tip Clearance on Tip Vortex in Axial Compressor. ASME J. Fluids Eng. 2006, 128, 1274-1280. [CrossRef]

41. Pelz, P.F.; Stonjek, S. Introduction of an universal scale-up method for the efficiency of axial and centrifugal fans. In Proceedings of the ASME Turbo Expo 2014: Turbine Conference and Exposition, Dusseldorf, Germany, 16-20 June 2014; ASME Paper No. GT2014-25403. p. V01AT10A007. [CrossRef]

42. Koch, C.C.; Smith, L.H. Loss sources and magnitudes in axial-flow compressor. ASME J. Gas. Turbines Power 1976, 98, 411-424. [CrossRef] 